TRANSACTIONS OF THE

AMERICAN MATHEMATICAL SOCIETY

Volume 364, Number 4, April 2012, Pages 1997-2019

S 0002-9947(2011)05390-0

Article electronically published on November 17, 2011

\title{
A CLASSIFICATION OF UNIPOTENT SPHERICAL CONJUGACY CLASSES IN BAD CHARACTERISTIC
}

\author{
MAURO COSTANTINI
}

\begin{abstract}
Let $G$ be a simple algebraic group over an algebraically closed field $k$ of bad characteristic. We classify the spherical unipotent conjugacy classes of $G$. We also show that if the characteristic of $k$ is 2 , then the fixed point subgroup of every involutory automorphism (involution) of $G$ is a spherical subgroup of $G$.
\end{abstract}

\section{INTRODUCTION}

Let $G$ be a simple algebraic group over an algebraically closed field $k$, with Lie algebra $\mathfrak{g}$. In this paper we determine the unipotent spherical conjugacy classes of $G$ (we recall that a conjugacy class $\mathcal{O}$ in $G$ is called spherical if a Borel subgroup of $G$ has a dense orbit on $\mathcal{O}$ ) when the characteristic of $k$ is bad for $G$. There has been much work related to this field in the cases of good characteristic.

To fix the notation, $B$ is a Borel subgroup of $G, T$ is a maximal torus of $B, B^{-}$is the Borel subgroup opposite to $B$, and $\left\{\alpha_{1}, \ldots, \alpha_{n}\right\}$ is the set of simple roots with respect to the choice of $(T, B)$. Let $W$ be the Weyl group of $G$ and let us denote by $s_{i}$ the reflection corresponding to the simple root $\alpha_{i}: \ell(w)$ is the length of the element $w \in W$ and $\operatorname{rk}(1-w)$ is the rank of $1-w$ in the geometric representation of $W$.

Initially, spherical $G$-orbits have been studied in the context of Lie algebras ([24], 25]) in characteristic zero. The classification of spherical nilpotent orbits has been obtained by Panyushev: in terms of height, a nilpotent orbit $\mathcal{O} \subset \mathfrak{g}$ is spherical if and only if its height is at most 3, which means 2 or 3 if $\mathcal{O}$ is not the zero orbit. Equivalently, $\mathcal{O}$ is spherical if and only if it contains an element of the form $e_{\gamma_{1}}+\cdots+e_{\gamma_{t}}$, where $\gamma_{1}, \ldots, \gamma_{t}$ are pairwise orthogonal simple roots (Panyushev 24], 26]).

More recently, in [5], we considered spherical conjugacy classes in $G$ over $\mathbb{C}$. We classify all spherical conjugacy classes by means of the Bruhat decomposition: a conjugacy class $\mathcal{O} \subset G$ is spherical if and only if $\operatorname{dim} \mathcal{O}=\ell(w)+\operatorname{rk}(1-w)$, where $w=w(\mathcal{O})$ is the unique element of $W$ such that $\mathcal{O} \cap B w B$ is open dense in $\mathcal{O}$ (we observe that the classification given in 5 over the complex numbers holds in general for characteristic zero).

In [15] the authors classify spherical nilpotent orbits in good characteristic using Kempf-Rousseau theory: the classification is the same as in the case of zero characteristic. In [8, the author obtains the classification of all spherical conjugacy classes in characteristic which is zero or is both good and odd by means of

Received by the editors January 1, 2010 and, in revised form, April 23, 2010 and May 28, 2010. 2010 Mathematics Subject Classification. Primary 20G15, 20E45.

(C)2011 American Mathematical Society 1997

Reverts to public domain 28 years from publication 
the Bruhat decomposition and by exploiting another characterization of spherical conjugacy classes available in such characteristic, namely a conjugacy class $\mathcal{O}$ is spherical if and only if $\{y \in W \mid \mathcal{O} \cap B y B \neq \emptyset\} \subseteq\left\{w \in W \mid w^{2}=1\right\}$ ([6. Theorem 2.7], [7, Theorem 5.7]).

In the present paper we complete the picture for unipotent spherical conjugacy classes by considering bad characteristic. Our strategy is to exhibit for each group $G$ a set $\mathcal{O}(G)$ of unipotent conjugacy classes, show that each element in $\mathcal{O}(G)$ is spherical, and finally show that each conjugacy class not in $\mathcal{O}(G)$ is not spherical. It turns out that in bad characteristic the classification of spherical unipotent conjugacy classes is the "same" as in zero characteristic, unless $p=2$ in type $C_{n}$ and $F_{4}$ or $p=3$ in type $G_{2}$. In these cases there are more classes than in characteristic zero. In particular, if $p=2$, then the conjugacy class of a non-trivial unipotent element $u$ is spherical if and only if $u$ is an involution.

It is well known that in zero or odd characteristic the fixed point subgroup $H$ of any involutory automorphism $\sigma$ of $G$ is a spherical subgroup (i.e. $G / H$ is a spherical homogeneous space). This was proved by Vust in 32 in characteristic zero (see also 23 over $\mathbb{C}$ ). Then Springer extended the result to odd characteristic in 29. In 27, Seitz gives an alternative proof of Springer's result. Here we prove that the result also holds in characteristic 2 .

The paper is structured as follows. In Section 2 we introduce the notation. In Section 3 we recall some basic facts about the classification of unipotent conjugacy classes in bad characteristic and determine the spherical ones. We also determine which spherical unipotent conjugacy classes have a representative $u$ of the form $u=$ $x_{\gamma_{1}}(1) \cdots x_{\gamma_{t}}(1)$, where $\gamma_{1}, \ldots, \gamma_{t}$ are pairwise orthogonal simple roots. In Section

4 we prove that if $G$ is a reductive connected algebraic group in characteristic 2 and $\sigma$ is any involutory automorphism of $G$, then the fixed point subgroup $H$ of $\sigma$ is a spherical subgroup of $G$.

\section{Preliminaries}

We denote by $\mathbb{C}$ the complex numbers, by $\mathbb{R}$ the reals, by $\mathbb{Z}$ the integers and by $\mathbb{N}$ the natural numbers.

Let $A=\left(a_{i j}\right)$ be a finite indecomposable Cartan matrix of rank $n$ with associated root system $\Phi$, and let $k$ be an algebraically closed field of characteristic char $k=p$. Let $G$ be a simple algebraic group over $k$ associated to $A$, with Lie algebra $\mathfrak{g}$. We fix a maximal torus $T$ of $G$ and a Borel subgroup $B$ containing $T: B^{-}$is the Borel subgroup opposite to $B$ and $U$ (respectively $U^{-}$) is the unipotent radical of $B$ (respectively of $B^{-}$). Then $\Phi$ is the set of roots relative to $T$ and $B$ determines the set of positive roots $\Phi^{+}$and the simple roots $\Delta=\left\{\alpha_{1}, \ldots, \alpha_{n}\right\}$. We fix a total ordering on $\Phi^{+}$compatible with the height function, and we shall write elements of $U$ as products of root elements where the roots are taken in the order given by this ordering. We shall use the numbering and the description of the simple roots in terms of the canonical basis $\left(e_{1}, \ldots, e_{k}\right)$ of an appropriate $\mathbb{R}^{k}$ as in 3 , Planches I-IX]. For the exceptional groups, we shall write $\beta=\left(m_{1}, \ldots, m_{n}\right)$ for $\beta=m_{1} \alpha_{1}+\cdots+m_{n} \alpha_{n}$. We denote by $P$ the weight lattice, by $P^{+}$the monoid of dominant weights and by $W$ the Weyl group. Also $s_{i}$ is the simple reflection associated to $\alpha_{i},\left\{\omega_{1}, \ldots, \omega_{n}\right\}$ are the fundamental weights, and $w_{0}$ is the longest element of $W$. The real space $E=\mathbb{R} P$ is a Euclidean space, endowed with the scalar product $\left(\alpha_{i}, \alpha_{j}\right)=d_{i} a_{i j}$. Here $\left\{d_{1}, \ldots, d_{n}\right\}$ are relatively prime positive 
integers such that if $D$ is the diagonal matrix with entries $d_{1}, \ldots, d_{n}$, then $D A$ is symmetric.

We put $\Pi=\{1, \ldots, n\}$ and fix a Chevalley basis $\left\{h_{i}, i \in \Pi ; e_{\alpha}, \alpha \in \Phi\right\}$ of $\mathfrak{g}$.

We use the notation $x_{\alpha}(\xi), h_{\alpha}(z)$, for $\alpha \in \Phi, \xi \in k, z \in k^{*}$ as in [31, 12]. For $\alpha \in \Phi$ we put $X_{\alpha}=\left\{x_{\alpha}(\xi) \mid \xi \in k\right\}$, the root subgroup corresponding to $\alpha$, and $H_{\alpha}=\left\{h_{\alpha}(z) \mid z \in k^{*}\right\}$. We identify $W$ with $N / T$, where $N$ is the normalizer of $T$ : given an element $w \in W$ we shall denote a representative of $w$ in $N$ by $\dot{w}$. We choose the $x_{\alpha}$ 's so that, for all $\alpha \in \Phi, n_{\alpha}=x_{\alpha}(1) x_{-\alpha}(-1) x_{\alpha}(1)$ lies in $N$ and has image the reflection $s_{\alpha}$ in $W$. Then

$$
x_{\alpha}(\xi) x_{-\alpha}\left(-\xi^{-1}\right) x_{\alpha}(\xi)=h_{\alpha}(\xi) n_{\alpha}, \quad n_{\alpha}^{2}=h_{\alpha}(-1)
$$

for every $\xi \in k^{*}, \alpha \in \Phi$ (30, Proposition 11.2.1]).

For algebraic groups we use the notation in [17, [13. In particular, for $J \subseteq \Pi$, $\Delta_{J}=\left\{\alpha_{j} \mid j \in J\right\}, \Phi_{J}$ is the corresponding root system, $W_{J}$ is the Weyl group, $P_{J}$ is the standard parabolic subgroup of $G$ and $L_{J}=T\left\langle X_{\alpha} \mid \alpha \in \Phi_{J}\right\rangle$ is the standard Levi subgroup of $P_{J}$. For $z \in W$ we put $U_{z}=U \cap z^{-1} U^{-} z$. Then the unipotent radical $R_{u} P_{J}$ of $P_{J}$ is $U_{w_{0} w_{J}}$, where $w_{J}$ is the longest element of $W_{J}$. Moreover, $U \cap L_{J}=U_{w_{J}}$ is a maximal unipotent subgroup of $L_{J}$.

If $\Psi$ is a subsystem of type $X_{r}$ of $\Phi$ and $H$ is the subgroup generated by $X_{\alpha}$, $\alpha \in \Psi$, we say that $H$ is an $X_{r}$-subgroup of $G$.

If $X$ is a $G$-variety and $x \in X$, we denote by $G . x$ the $G$-orbit of $x$ and by $G_{x}$ the isotropy subgroup of $x$ in $G$. If the homogeneous space $G / H$ is spherical, we say that $H$ is a spherical subgroup of $G$.

If $x$ is an element of a group $K$ we denote by $\mathcal{O}_{x}$ its conjugacy class and by $C(x)$ its centralizer. If $H \leq K$, we shall denote by $C_{H}(x)$ the centralizer of $x$ in $H$. If $x, y \in K$, then $x \sim y$ means that $x, y$ are conjugate in $K$. For unipotent classes in exceptional groups we use the notation in [13. We use the description of centralizers of involutions as in [18, 22.

We denote by $\mathbb{Z}_{r}$ the cyclic group of order $r$.

For each conjugacy class $\mathcal{O}$ in $G, w=w(\mathcal{O})$ is the unique element of $W$ such that $B w B \cap \mathcal{O}$ is open dense in $\mathcal{O}$. We shall also say that $\mathcal{O}$ lies over $w$.

In the remainder of the paper we shall denote by $p$ the characteristic of $k$ (hence $p$ may be 0$)$.

\section{The CLASSIFICATION}

We recall that the bad primes for the individual types of simple groups are as follows:

none when $G$ has type $A_{n}$;

$p=2$ when $G$ has types $B_{n}, C_{n}, D_{n}$;

$p=2$ or 3 when $G$ has types $G_{2}, F_{4}, E_{6}, E_{7}$;

$p=2,3$ or 5 when $G$ has type $E_{8}$.

One may find a detailed account of the classification of both unipotent classes and nilpotent orbits in bad characteristic in [13, §5.11].

Strategy of the proof. Let $G_{\mathbb{C}}$ be the corresponding group over $\mathbb{C}$. We have shown in [5] that for every spherical conjugacy class $\mathcal{C}$ of $G_{\mathbb{C}}$ there exists an involution $w=w(\mathcal{C})$ in $W$ such that $\operatorname{dim} \mathcal{C}=\ell(w)+\operatorname{rk}(1-w)$, with $\mathcal{C} \cap B w B \neq \emptyset$. For each group $G$ we introduce a certain set $\mathcal{O}(G)$ of unipotent conjugacy classes which are 
candidates for being spherical. For each $\mathcal{O} \in \mathcal{O}(G)$ we show that there is a (not necessarily unipotent) spherical conjugacy class $\mathcal{C}$ in $G_{\mathbb{C}}$ such that

$$
\operatorname{dim} \mathcal{O}=\operatorname{dim} \mathcal{C} .
$$

Let $w=w(\mathcal{C})$. Our aim is to show that $\mathcal{O} \cap B w B \neq \emptyset$.

Definition 3.1. Let $\mathcal{O}$ be a conjugacy class of $G$. We say that $\mathcal{O}$ satisfies $(*)$ if there exists $w \in W$ such that $B w B \cap \mathcal{O} \neq \emptyset$ and $\operatorname{dim} \mathcal{O}=\ell(w)+\operatorname{rk}(1-w)$.

Let $\mathcal{O}$ be a conjugacy class in $G$. There exists a unique element $w=w(\mathcal{O}) \in W$ such that $\mathcal{O} \cap B w B$ is open dense in $\mathcal{O}$. In particular,

$$
\overline{\mathcal{O}}=\overline{\mathcal{O} \cap B w B} \subseteq \overline{B w B} .
$$

It follows that if $y$ is an element of $\overline{\mathcal{O}}$ and $y \in B z B$, then $z \leq w$ in the ChevalleyBruhat order of $W$.

We recall the following result proved in [5, Theorem 5] over $\mathbb{C}$, but which is valid with the same proof over any algebraically closed field.

Theorem 3.2. Suppose that $\mathcal{O}$ contains an element $x \in B w B$. Then

$$
\operatorname{dim} B . x \geq \ell(w)+\operatorname{rk}(1-w) .
$$

In particular, $\operatorname{dim} \mathcal{O} \geq \ell(w)+\operatorname{rk}(1-w)$. If, in addition, $\operatorname{dim} \mathcal{O} \leq \ell(w)+\operatorname{rk}(1-w)$, then $\mathcal{O}$ is spherical, $w=w(\mathcal{O})$ and B.x is the dense B-orbit in $\mathcal{O}$.

Let $\mathcal{O}$ be a conjugacy class of $G$ and let $w=w(\mathcal{O})$. If $\mathcal{O}^{-1}=\mathcal{O}$ (i.e. if any element $x \in \mathcal{O}$ is conjugate to its inverse), then $w^{2}=1$. It is well known that over any algebraically closed field any unipotent element is conjugate to its inverse ([9. Lemma 1.16], [10, Lemma 2.3]; see also [22, Proposition 2.5 (a)]) so that $w$ is an involution for every non-trivial unipotent conjugacy class $\mathcal{O}$. However, it has recently been shown in 14 that $w^{2}=1$ for every conjugacy class $\mathcal{O}$ in $G$.

If $g$ is in $Z(G)$, then $g \in T$, its conjugacy class $\mathcal{O}$ is simply $\{g\}$, and $w(\mathcal{O})=1$. In the remainder of the paper we shall consider only non-central conjugacy classes.

We shall use the following result.

Lemma 3.3. Assume the positive roots $\beta_{1}, \ldots, \beta_{\ell}$ are such that $\left[X_{ \pm \beta_{i}}, X_{ \pm \beta_{j}}\right]=1$ for every $1 \leq i<j \leq \ell$. Then, for $\xi_{1}, \ldots, \xi_{\ell} \in k^{*}, g=x_{\beta_{1}}\left(-\xi_{1}^{-1}\right) \cdots x_{\beta_{\ell}}\left(-\xi_{\ell}^{-1}\right)$, and $h=h_{\beta_{1}}\left(-\xi_{1}\right) \cdots h_{\beta_{\ell}}\left(-\xi_{\ell}\right)$ we have

$$
g x_{-\beta_{1}}\left(\xi_{1}\right) \cdots x_{-\beta_{\ell}}\left(\xi_{\ell}\right) g^{-1}=n_{\beta_{1}} \cdots n_{\beta_{\ell}} h x_{\beta_{1}}\left(2 \xi_{1}^{-1}\right) \cdots x_{\beta_{\ell}}\left(2 \xi_{\ell}^{-1}\right) .
$$

Proof. By (2.1) we have $x_{\alpha}\left(-\xi^{-1}\right) x_{-\alpha}(\xi) x_{\alpha}\left(\xi^{-1}\right)=n_{\alpha} h_{\alpha}(-\xi) x_{\alpha}\left(2 \xi^{-1}\right)$. The result follows from $\left[X_{ \pm \beta_{i}}, X_{ \pm \beta_{j}}\right]=1$ for every $1 \leq i<j \leq \ell$.

The hypothesis of the lemma is satisfied, for instance, if $\beta_{1}, \ldots, \beta_{\ell}$ are pairwise orthogonal and long, as in [11, Lemma 4.1]. In characteristic 2 , we have $\left[X_{\gamma}, X_{\delta}\right]=1$ for every pair $(\gamma, \delta)$ of orthogonal roots, so that for any set of pairwise orthogonal roots $\beta_{1}, \ldots, \beta_{\ell}$ and for $g=x_{\beta_{1}}(1) \cdots x_{\beta_{\ell}}(1)$ we get

$$
g x_{-\beta_{1}}(1) \cdots x_{-\beta_{\ell}}(1) g^{-1}=n_{\beta_{1}} \cdots n_{\beta_{\ell}} .
$$

We shall prove the following theorem.

Theorem 3.4. Let $\mathcal{O}$ be a non-trivial unipotent conjugacy class of a simple algebraic group in characteristic 2 . Then $\mathcal{O}$ is spherical if and only if it consists of involutions. 
From the classification it will follow that the unipotent conjugacy class $\mathcal{O}$ is spherical if and only if it satisfies $(*)$. Moreover, we shall see that there is a unique maximal spherical conjugacy class $\mathcal{O}_{\max }$ and that it has the following properties: $w\left(\mathcal{O}_{\max }\right)=w_{0}$, and the closure of $\mathcal{O}_{\max }$ is the union of all spherical unipotent orbits.

3.1. Classical groups in characteristic 2. To deal with the classical groups with $p=2$, we recall that the unipotent classes were determined by Wall in 33] (the nilpotent orbits were determined by Hesselink in [16]). For the convenience of the reader, here we recall the classification of unipotent classes in the classical groups following [28, §2]. Suppose $G=G L(n)$ (any characteristic) and $u$ is a unipotent element of $G$. Then one can associate to $u$ a partition $\lambda=\left(\lambda_{1}, \lambda_{2}, \ldots\right)=$ $1^{c(1)} \oplus 2^{c(2)} \oplus \cdots$ of $n$ with $\lambda_{1} \geq \lambda_{2} \geq \cdots$, where $c(i)$ is the number of Jordan blocks of $u$ of dimension $i$, for every $i \geq 1$. In this way the set $\mathcal{C} U(G)$ of unipotent conjugacy classes of $G$ is parametrized by the set of partitions of $n$. We denote by $C_{\lambda}$ the unipotent class corresponding to the partition $\lambda$. The set $\mathcal{C} U(G)$ has a natural partial order: $\mathcal{O}_{1} \leq \mathcal{O} \Leftrightarrow \mathcal{O}_{1} \subseteq \overline{\mathcal{O}}$. If we partially order the set of partitions of $n$ by $\lambda \leq \mu \Leftrightarrow \sum_{j=1}^{i} \lambda_{j} \leq \sum_{j=1}^{i} \mu_{j}$ for every $i \geq 1$, then the map $\lambda \mapsto C_{\lambda}$ is an isomorphism of p.o. sets.

Now assume $p=2$. In this case there exists a homomorphism (central isogeny) of $S O(2 n+1)$ onto $S p(2 n)$ which is an isomorphism of abstract groups. We shall therefore deal only with $S p(2 n)$ and $S O(2 n)$. Let $\omega$ be an object distinct from 0 and 1 , and consider the set $\{\omega, 0,1\}$ totally ordered by $\omega<0<1$. Assume $G=S p(2 n) \leq G L(2 n)$ (resp. $G=O(2 n) \leq S p(2 n)$ ). The unipotent conjugacy classes of $G$ are parametrized by pairs $(\lambda, \varepsilon)$ such that

a) $\lambda=1^{c(1)} \oplus 2^{c(2)} \oplus \cdots$ is a partition of $2 n$ with $c(i)$ even for every odd $i$.

b) $\varepsilon: \mathbb{N} \rightarrow\{\omega, 0,1\}$ is such that

$\left.\mathrm{b}_{1}\right) \varepsilon(i)=\omega$ if $i$ is odd or $i \geq 1$ and $c(i)=0$.

$\left.\mathrm{b}_{2}\right) \varepsilon(i)=1$ if $i$ is even and $c(i)$ is odd $(i \neq 0)$.

$\left.\mathrm{b}_{3}\right) \varepsilon(i) \neq \omega$ if $i$ is even and $c(i) \neq 0(i \neq 0)$.

$\left.\mathrm{b}_{4}\right) \varepsilon(0)=1$ (resp. $\left.\varepsilon(0)=0\right)$.

The correspondence is obtained as follows. Let $u$ be a unipotent element of $G$. Then $u$ determines a class in $G L(2 n)$, hence the partition $\lambda$ of $2 n$. This partition satisfies a). Now, if $i$ is even, $i \neq 0$ and $c(i) \neq 0$, we put $\varepsilon(i)=0$ if

$$
f\left((u-1)^{i-1}(x), x\right)=0
$$

for every $x \in \operatorname{ker}(u-1)^{i}$, and $\varepsilon(i)=1$ otherwise (here $f$ is the bilinear form used to define $S p(2 n)$ ). In view of condition b), this uniquely defines $\varepsilon$. For $i \in \mathbb{N}$ we shall write $c_{i}$ and $\varepsilon_{i}$ for $c(i)$ and $\varepsilon(i)$.

We denote by $C_{\lambda, \varepsilon}$ the unipotent class of $G$ corresponding to $(\lambda, \varepsilon)$. We observe that every unipotent class of $S p(2 n)$ intersects $O(2 n)$ in a unique class of $O(2 n)$. Moreover, the unipotent classes of $O(2 n)$ contained in $S O(2 n)$ (the connected component of $O(2 n))$ are those for which $\lambda_{1}^{*}$ is even $\left(\lambda^{*}\right.$ is the dual partition of $\left.\lambda\right)$. If all $\lambda_{i}$ 's and $c(i)$ 's are even and if $\varepsilon(i) \neq 1$ for every $i$, then the unipotent class $C_{\lambda, \varepsilon}$ of $O(2 n)$ splits into two classes of $S O(2 n)$. All the other unipotent classes in $S O(2 n)$ are unipotent classes in $O(2 n)$.

We shall add the subscript 0 in the case when $i$ is even, $c(i)$ is even and $\varepsilon(i)=0$.

In [28, $\S 2.8,2.9,2.10]$, there are formulas for the dimensions of centralizers of unipotent elements in $S p(2 n), O(2 n)$ (hence also in $S O(2 n)$ ), the determination of 
TABle 1. Spherical unipotent classes in $A_{n}, p=2$.

\begin{tabular}{|c||c|c|c|c|}
\hline $\mathcal{O}$ & $J$ & $w(\mathcal{O})$ & $x \in \mathcal{O} \cap B w(\mathcal{O}) B$ & $\operatorname{dim} \mathcal{O}$ \\
\hline \hline $\begin{array}{c}X_{\ell}=2^{\ell} \oplus 1^{n+1-2 \ell} \\
\ell=1, \ldots,\left[\frac{n+1}{2}\right]\end{array}$ & $J_{\ell}$ & $s_{\beta_{1}} \cdots s_{\beta_{\ell}}$ & $n_{\beta_{1}} \cdots n_{\beta_{\ell}}$ & $2 \ell(n+1-\ell)$ \\
\hline
\end{tabular}

the component groups $C(u) / C(u)^{\circ}$ in the various cases, and an explicit definition of a partial order on pairs $(\lambda, \varepsilon)$ such that $C_{\lambda, \varepsilon} \leq C_{\mu, \phi} \Leftrightarrow(\lambda, \varepsilon) \leq(\mu, \phi)$.

We shall use the notation as in 28. As mentioned above, we need only treat $S p(2 n)$ and $S O(2 n)$. However, for completeness, we shall also deal with the case when $G$ has type $A_{n}$, since this is dealt with in [15] but not in [8].

3.1.1. Type $A_{n}, n \geq 1$. We show that every spherical unipotent conjugacy class satisfies $(*)$. The spherical nilpotent orbits (and therefore the spherical unipotent classes) have been classified in [15, and it follows that a unipotent conjugacy class $\mathcal{O}$ is spherical if and only if $\mathcal{O}=X_{\ell}$, the unipotent class $2^{\ell} \oplus 1^{n+1-2 \ell}$ for $\ell=$ $1, \ldots,\left[\frac{n+1}{2}\right]$. For every $i=1, \ldots,\left[\frac{n+1}{2}\right]$, let $\beta_{i}=e_{i}-e_{n+2-i}$. Then, as for $\mathbb{C}$ (and for any algebraically closed field of odd or zero characteristic) the element $u=$ $x_{-\beta_{1}}(1) \cdots x_{-\beta_{\ell}}(1)$ lies in $X_{\ell} \cap B w B$, where $w=s_{\beta_{1}} \cdots s_{\beta_{\ell}}$ is such that $\operatorname{dim} X_{\ell}=$ $\ell(w)+\operatorname{rk}(1-w)$. In fact one may take $n_{\beta_{1}} \cdots n_{\beta_{\ell}} \in s_{\beta_{1}} \cdots s_{\beta_{\ell}} B \cap X_{\ell}$. For $\ell=$ $1, \ldots,\left[\frac{n+1}{2}\right]-1$ we put $J_{\ell}=\{\ell+1, \ldots, n-\ell\}, J_{\left[\frac{n+1}{2}\right]}=\varnothing$. We have established the information presented in Table 1, where $w(\mathcal{O})=w_{0} w_{J}$.

Proposition 3.5. Let $\mathcal{O}$ be a non-trivial unipotent conjugacy class of $S L(n+1)$ in characteristic 2 . Then $\mathcal{O}$ is spherical if and only if it consists of involutions if and only if $\mathcal{O}$ has a representative of the form $x_{\gamma_{1}}(1) \cdots x_{\gamma_{t}}(1)$, where $\gamma_{1}, \ldots, \gamma_{t}$ are pairwise orthogonal simple roots. All non-trivial spherical unipotent classes are listed in Table 1

3.1.2. Type $C_{n}$ (and $B_{n}$ ), $n \geq 2$. We first show that if $u$ is an involution of $G$, then $\mathcal{O}_{u}$ is spherical, by showing that $\mathcal{O}_{u}$ satisfies $(*)$. Therefore, let $u$ be an involution of $G=S p(2 n)$. Then the partition corresponding to $u$ is of the form $\lambda=1^{c_{1}} \oplus 2^{c_{2}}$, with $c_{2}=\ell, c_{1}=2 n-2 \ell, \ell=1, \ldots, n$.

Using the description of unipotent conjugacy classes recalled above, let $\lambda=$ $2^{\ell} \oplus 1^{2 n-2 \ell}$, for $\ell=1, \ldots, n$. Then we have $\varepsilon_{0}=1, \varepsilon_{1}=\omega, \varepsilon_{i}=\omega$, for $i \geq 3$. As for $\varepsilon_{2}$, we have $\varepsilon_{2}=1$ if $\ell$ is odd. On the other hand, if $\ell$ is even, we have both possibilities $\varepsilon_{2}=0$ or 1 . We denote by $X_{\ell}$ the class corresponding to $\varepsilon_{2}=1$ and by $Y_{\ell}$ the class corresponding to $\varepsilon_{2}=0$ (when $\ell$ is even).

We denote by $X_{\ell, \mathbb{C}}$ the unipotent class in $\operatorname{Sp}(2 n, \mathbb{C})$ corresponding to $\lambda=2^{\ell} \oplus$ $1^{2 n-2 \ell}$. Then we get

$$
\begin{array}{lll}
\operatorname{dim} X_{\ell}=\operatorname{dim} X_{\ell, \mathbb{C}} & =\ell(2 n-\ell+1) & \ell \in\{1, \ldots, n\}, \\
\operatorname{dim} Y_{\ell}=\operatorname{dim} X_{\ell, \mathbb{C}}-\ell & =\ell(2 n-\ell) & \ell \in\{1, \ldots, n\}, \ell \text { even. }
\end{array}
$$

Note that if $\ell$ is even and we write $\ell=2 \ell^{\prime}$, then $\operatorname{dim} Y_{\ell}=\operatorname{dim} \mathcal{O}_{\sigma_{\ell^{\prime}}, \mathbb{C}}$, where $\mathcal{O}_{\sigma_{\ell^{\prime}}, \mathbb{C}}$ is the conjugacy class in $S p(2 n, \mathbb{C})$ of the involution $\sigma_{\ell^{\prime}}([5$, Table 1]). In $S p(2 n, \mathbb{C})$, the spherical semisimple conjugacy class $\mathcal{O}_{\sigma_{\ell^{\prime}, \mathbb{C}}}$ lies over $w=s_{\gamma_{1}} \cdots s_{\gamma_{\ell^{\prime}}}$ ([5, Table 5], [11, §4.2.2]). 
We observe that if the partition associated to the involution $u$ is $\lambda=2^{2 \ell} \oplus 1^{2 n-4 \ell}$, then $\mathcal{O}_{u}=2_{0}^{2 \ell} \oplus 1^{2 n-4 \ell}$ if and only if $f((u-1) v, v)=0$ for every $v \in V$ (here $f$ is the bilinear form on $V$ used to define $\left.S p_{2 n}(k)\right)$. Let $w$ be an involution of $W, L(w)=$ $\left\{\beta \in \Phi^{+} \mid w(\beta)=-\beta, \beta\right.$ long $\}$, and $L(w)_{\perp}=\left\{\gamma \in \Phi^{+} \mid w(\gamma)=-\gamma,(\gamma, L(w))=\right.$ $0, \gamma$ short $\}$. Then $w=\prod_{\beta \in L(w)} s_{\beta} \prod_{\gamma \in L(w)_{\perp}} s_{\gamma}$. Let $x=\prod_{\beta \in L(w)} n_{\beta} \prod_{\gamma \in L(w)_{\perp}} n_{\gamma}$. Then $x$ is an involution in $B w B$ and the number of blocks of dimension 2 in the Jordan canonical form of $x$ is $|L(w)+2| L(w)_{\perp}$. If this number is even, then $f((x-1) v, v)=0$ for every $v \in V$ if and only if $L(w)=\emptyset$.

We put $\beta_{i}=2 e_{i}$ for $i=1, \ldots, n$ and $\gamma_{i}=e_{2 i-1}+e_{2 i}$ for $i=1, \ldots,\left[\frac{n}{2}\right]$.

Then it is straightforward to show that

$$
\begin{array}{ll}
x_{-\beta_{1}}(1) \cdots x_{-\beta_{\ell}}(1) \in X_{\ell} \cap B s_{\beta_{1}} \cdots s_{\beta_{\ell}} B \cap U^{-} & \text {for } \ell=1, \ldots, n, \\
x_{-\gamma_{1}}(1) \cdots x_{-\gamma_{\ell}}(1) \in Y_{2 \ell} \cap B s_{\gamma_{1}} \cdots s_{\gamma_{\ell}} B \cap U^{-} & \text {for } \ell=1, \ldots,\left[\frac{n}{2}\right] .
\end{array}
$$

By (3.2), we can choose

$$
\begin{gathered}
n_{\beta_{1}} \cdots n_{\beta_{\ell}} \in X_{\ell} \cap w B \quad \text { for } \ell=1, \ldots, n, \\
n_{\gamma_{1}} \cdots n_{\gamma_{\ell}} \in Y_{2 \ell} \cap w B \quad \text { for } \ell=1, \ldots,\left[\frac{n}{2}\right] .
\end{gathered}
$$

One can easily deduce which classes of involutions have a representative of the form $u=\prod_{i \in K} x_{\alpha_{i}}(1)$ for a certain subset $K$ of $\Pi$. Note that since $u$ is an involution, $\left(\alpha_{i}, \alpha_{j}\right)=0$ if $i, j \in K$ with $i \neq j$. Up to the $W$ action, we have only the following representatives $u$ :

$$
\begin{gathered}
\prod_{i=1}^{\ell} x_{\alpha_{n-2(i-1)}}(1) \in X_{2 \ell-1} \quad \text { for } \ell=1, \ldots,\left[\frac{n+1}{2}\right] \\
\prod_{i=1}^{\ell} x_{\alpha_{2 i-1}}(1) \in Y_{2 \ell} \quad \text { for } \ell=1, \ldots,\left[\frac{n}{2}\right] .
\end{gathered}
$$

These exhaust the conjugacy classes of involutions with representative of the form $\prod_{i \in K} x_{\alpha_{i}}(1)$. In particular, all $X_{2 \ell}$ have no representative of the form $\prod_{i \in K} x_{\alpha_{i}}(1)$. The point is that in good characteristic, for $\ell=1, \ldots,\left[\frac{n}{2}\right]$ the element $\prod_{i=1}^{\ell} x_{\alpha_{2 i-1}}(1)$ is conjugate to $\prod_{i=1}^{2 \ell} x_{\beta_{i}}(1)$ (which lies in $X_{2 \ell}$ ).

By Theorem 3.2, we have proved

Proposition 3.6. Let $\mathcal{O}$ be the conjugacy class of an involution of $S p(2 n)$ in characteristic 2 . Then $\mathcal{O}$ is spherical.

Our aim is to show that a (non-trivial) unipotent conjugacy class $\mathcal{O}_{u}$ is spherical if and only if $u$ is an involution. By [15, Remark 2.14], the orbit $\mathcal{O}$ is spherical if and only if $G / H$ is spherical, where $H$ is the isotropy subgroup of an element in $\mathcal{O}$. Moreover, $G / H$ is spherical if and only if $G / H^{\circ}$ is spherical, where $H^{\circ}$ denotes the connected component of $H$. We shall therefore use the following.

Lemma 3.7. Let $\mathcal{O}$ be a $G$-orbit with isotropy subgroup $H$. Then $\mathcal{O}$ is spherical if and only if $G / H^{\circ}$ is spherical.

By Proposition 3.6, we are left to show that if the (non-trivial) unipotent class $\mathcal{O}$ does not consist of involutions, then $\mathcal{O}$ is not spherical. Let $u$ be a unipotent element of order greater than 4 , and let $v$ be an element of order 4 in the subgroup generated by $u$. Since $C(u) \leq C(v)$, if $\mathcal{O}_{v}$ is non-spherical, then $\mathcal{O}_{u}$ is also non-spherical. We 
are therefore left to consider the set $X$ of conjugacy classes of elements of order 4 . By [19, Theorem 2.2], it is enough to show that the minimal elements in $X$ are not spherical.

From the explicit definition of a partial order on pairs $(\lambda, \varepsilon)$ such that $C_{\lambda, \varepsilon} \leq$ $C_{\mu, \phi} \Leftrightarrow(\lambda, \varepsilon) \leq(\mu, \phi)$ given in [28, §2.10], it follows that the minimal elements in $X$ are the classes $3^{2} \oplus 1^{2 n-6}$ (if $n \geq 3$ ) and $4 \oplus 1^{2 n-4}$.

In the following lemmas we deal with these cases. We also consider a case in $D_{n}$.

Lemma 3.8. Let $\mathcal{O}$ be the unipotent conjugacy class of type $3^{2} \oplus 1^{2 n-6}$ in $C_{n}$ or $D_{n}$. Then $\mathcal{O}$ is not spherical $(p=2)$.

Proof. Let $u$ be an element in $\mathcal{O}=3^{2} \oplus 1^{2 n-6}$ (this exists in $C_{n}$ if $n \geq 3$ ). In $S p(2 n)$, we may take $u=x_{\alpha_{2}}(1) x_{\gamma-\alpha_{2}}(1)$, where $\gamma$ is the highest short root $\left(\gamma=e_{1}+e_{2}\right)$, and get $C(u)^{\circ} \leq P$, where $P$ is the maximal parabolic subgroup $P_{I \backslash\left\{\alpha_{2}\right\}}$. Then $C(u)^{\circ}=C R, C=H_{\alpha_{1}} \times K$, where $K$ is the $C_{n-3}$-subgroup of $G$ corresponding to $\left\{\alpha_{4}, \ldots, \alpha_{n}\right\}$, and $R$ is the subgroup

$$
\left\{u \in R_{u} P\left|u=\prod_{\alpha \in \Phi^{+}} x_{\alpha}\left(z_{\alpha}\right)\right| z_{\alpha_{2}}=z_{\gamma-\alpha_{2}}\right\}
$$

of codimension 1 in $R_{u} P$. It follows that $C(u)^{\circ}$ fixes the element $e_{\alpha_{2}}+e_{\gamma-\alpha_{2}}$ of $\mathfrak{g}$. However, we clearly have $C(u) \leq C\left(u^{2}\right)$, and $u^{2}=x_{\gamma}(1)$. But $C\left(x_{\gamma}(1)\right)$ fixes the element $e_{\gamma}$ of $\mathfrak{g}$, since $x_{\gamma}(1)=1+e_{\gamma}$ in $M_{2 n}(k)$. It follows that $C(u)^{\circ}$ has 2 linearly independent invariants in $\mathfrak{g}$ so that we can find linearly independent functions $f$, $g$ in $k\left[S p(2 n) / C(u)^{\circ}\right]$ which are $B$-weight vectors of the same weight. Then $f / g$ is a non-constant $B$-invariant rational function on $S p(2 n) / C(u)^{\circ}$, which is therefore not spherical by Rosenlicht's Theorem (see [4, Lemma 1.1]).

Since $u$ lies in $S O(2 n)$ and both $e_{\alpha_{2}}+e_{\gamma-\alpha_{2}}$ and $e_{\gamma}$ are in the Lie algebra of $S O(2 n)$, the $S O(2 n)$-orbit of $u$ is not spherical as well.

Lemma 3.9. Let $\mathcal{O}$ be the unipotent conjugacy class of type $4 \oplus 1^{2 n-4}$ in $C_{n}$. Then $\mathcal{O}$ is not spherical $(p=2)$.

Proof. Let $u$ be an element in $\mathcal{O}=4 \oplus 1^{2 n-4}$. In $S p(2 n)$, we may take $u=$ $x_{\alpha_{1}}(1) x_{\delta}(1)$, where $\delta=2 e_{2}$, and get $C(u)^{\circ} \leq P$, where $P$ is the parabolic subgroup $P_{I \backslash\left\{\alpha_{1}, \alpha_{2}\right\}}$. Then $C(u)^{\circ}=C R$, where $C$ is the $C_{n-2^{-}}$-subgroup of $G$ corresponding to $\left\{\alpha_{3}, \ldots, \alpha_{n}\right\}$, and $R$ is a subgroup of $U$. In fact $\operatorname{dim} R=2 n-2$, and $R$ is the product of $X_{\alpha}$ 's, where $\alpha=e_{1} \pm e_{i}, i=3, \ldots, n$, or $\alpha=e_{1}+e_{2}$ or $\alpha=2 e_{1}$.

It follows that $C(u)^{\circ}$ fixes the first 2 basis vectors $v_{1}$ and $v_{2}$ of the natural module of $G$ so that $S p(2 n) / C(u)^{\circ}$ is not spherical, as in the previous case.

If $J_{\ell}=\{\ell+1, \ldots, n\}\left(J_{n}=\varnothing\right)$ for $\ell=1, \ldots, n, K_{\ell}=\{1,3, \ldots, 2 \ell-1,2 \ell+$ $1,2 \ell+2, \ldots, n\}$ for $\ell=1, \ldots,\left[\frac{n}{2}\right]$, we have established the information presented in Table 2, where $w(\mathcal{O})=w_{0} w_{J}$.

We have proved

Proposition 3.10. Let $\mathcal{O}$ be a non-trivial unipotent conjugacy class of $S p(2 n)$ in characteristic 2. Then $\mathcal{O}$ is spherical if and only if it consists of involutions. All non-trivial spherical unipotent classes are listed in Table 2 .

3.1.3. Type $D_{n}, n \geq 4$. Let $m=\left[\frac{n}{2}\right]$. We put $\beta_{i}=e_{2 i-1}+e_{2 i}, \delta_{i}=e_{2 i-1}-e_{2 i}$ for $i=1, \ldots, m$. For $\ell=1, \ldots, m-1$ we put $J_{\ell}=\{2 \ell+1, \ldots, n\}, J_{m}=\varnothing$; $K_{\ell}=J_{\ell} \cup\{1,3, \ldots, 2 \ell-1\}$ for $\ell=1, \ldots, m$. Also, if $n$ is even, we put $K_{m}^{\prime}=$ $\{1,3, \ldots, n-3, n\}$. 
TABLE 2. Spherical unipotent classes in $C_{n}, n \geq 2, p=2$.

\begin{tabular}{|c||c|c|c|c|}
\hline $\mathcal{O}$ & $J$ & $w(\mathcal{O})$ & $x \in \mathcal{O} \cap B w(\mathcal{O}) B$ & $\operatorname{dim} \mathcal{O}$ \\
\hline \hline $\begin{array}{c}X_{\ell}=2^{\ell} \oplus 1^{2 n-2 \ell} \\
\ell=1, \ldots, n\end{array}$ & $J_{\ell}$ & $s_{\beta_{1}} \cdots s_{\beta_{\ell}}$ & $n_{\beta_{1}} \cdots n_{\beta_{\ell}}$ & $\ell(2 n-\ell+1)$ \\
\hline $\begin{array}{c}Y_{2 \ell}=2_{0}^{2 \ell} \oplus 1^{2 n-4 \ell} \\
\ell=1, \ldots,\left[\frac{n}{2}\right]\end{array}$ & $K_{\ell}$ & $s_{\gamma_{1}} \cdots s_{\gamma_{\ell}}$ & $n_{\gamma_{1}} \cdots n_{\gamma_{\ell}}$ & $4 \ell(n-\ell)$ \\
\hline
\end{tabular}

Let $u$ be an involution of $G=S O(2 n)$. Then the partition corresponding to $u$ is of the form $\lambda=2^{c_{2}} \oplus 1^{c_{1}}$, with $c_{2}=2 \ell, c_{1}=2 n-4 \ell, \ell=1, \ldots, m$.

For each $\ell=1, \ldots, m-1$ there are 2 conjugacy classes corresponding to $\lambda=$ $2^{2 \ell} \oplus 1^{2 n-4 \ell}$ : we denote by $X_{\ell}$ the class $2_{0}^{2 \ell} \oplus 1^{2 n-4 \ell}$ and by $Z_{\ell}$ the class $2^{2 \ell} \oplus 1^{2 n-4 \ell}$. If $\ell=m$, then we denote by $Z_{m}$ the class $2^{2 m} \oplus 1^{2 n-4 m}$. The conjugacy class in $O(2 n)$ corresponding to $2_{0}^{2 m} \oplus 1^{2 n-4 m}$ is a single class $X_{m}$ in $S O(2 n)$ if $n$ is odd, while it splits into 2 conjugacy classes $X_{m}$ and $X_{m}^{\prime}$ in $S O(2 n)$ if $n$ is even.

We have

$$
\operatorname{dim} Z_{\ell}=4 \ell(n-\ell), \quad \operatorname{dim} X_{\ell}=2 \ell(2 n-2 \ell-1) \quad \text { for } \ell=1, \ldots, m,
$$

with $\operatorname{dim} X_{m}^{\prime}=\operatorname{dim} X_{m}$ if $n$ is even. We have chosen the notation so that for each conjugacy class of involutions $\mathcal{O}$ in $G$, the conjugacy class $\mathcal{C}$ in $G_{\mathbb{C}}$ denoted by the same symbol in [11, §4.3] has the same dimension. For the corresponding $w$, we write $w$ as a product of commuting reflections, $w=s_{\gamma_{1}} \cdots s_{\gamma_{t}}$. It is straightforward to prove that in each case the element $x=n_{\gamma_{1}} \cdots n_{\gamma_{t}}$ lies in $\mathcal{O}$. By Theorem 3.2, we have proved

Proposition 3.11. Let $\mathcal{O}$ be the conjugacy class of an involution of $S O(2 n)$ in characteristic 2. Then $\mathcal{O}$ is spherical.

Our aim is to show that a (non-trivial) unipotent conjugacy class $\mathcal{O}_{u}$ is spherical if and only if $u$ is an involution. Using the same arguments as in case $C_{n}$, we are left to consider the set $X$ of conjugacy classes of elements of order 4 , and then show that the minimal elements in $X$ are not spherical. From the explicit definition of a partial order on pairs $(\lambda, \varepsilon)$ such that $C_{\lambda, \varepsilon} \leq C_{\mu, \phi} \Leftrightarrow(\lambda, \varepsilon) \leq(\mu, \phi)$ given in 28, $\S 2.10]$, it follows that the minimal element in $X$ is the class $3^{2} \oplus 1^{2 n-6}$. By Lemma 3.8, this class is not spherical.

We have established the information presented in Tables 3 and 4 , where $w(\mathcal{O})=$ $w_{0} w_{J}$.

TABLE 3. Spherical unipotent classes in $D_{n}, n \geq 4, n=2 m, p=2$.

\begin{tabular}{|c||c|c|c|c|}
\hline $\mathcal{O}$ & $J$ & $w(\mathcal{O})$ & $x \in \mathcal{O} \cap B w(\mathcal{O}) B$ & $\operatorname{dim} \mathcal{O}$ \\
\hline \hline $\begin{array}{c}Z_{\ell}=2^{2 \ell} \oplus 1^{2 n-4 \ell} \\
\ell=1, \ldots, m\end{array}$ & $J_{\ell}$ & $s_{\beta_{1}} s_{\delta_{1}} \cdots s_{\beta_{\ell}} s_{\delta_{\ell}}$ & $n_{\beta_{1}} n_{\delta_{1}} \cdots n_{\beta_{\ell}} n_{\delta_{\ell}}$ & $4 \ell(n-\ell)$ \\
\hline $\begin{array}{c}X_{\ell}=2_{0}^{2 \ell} \oplus 1^{2 n-4 \ell} \\
\ell=1, \ldots, m\end{array}$ & $K_{\ell}$ & $s_{\beta_{1}} \cdots s_{\beta_{\ell}}$ & $n_{\beta_{1}} \cdots n_{\beta_{\ell}}$ & $2 \ell(2 n-2 \ell-1)$ \\
\hline$X_{m}^{\prime}=\left(2_{0}^{2 m}\right)^{\prime}$ & $K_{m}^{\prime}$ & $s_{\beta_{1}} \cdots s_{\beta_{m-1}} s_{\alpha_{n-1}}$ & $n_{\beta_{1}} \cdots n_{\beta_{m-1}} n_{\alpha_{n-1}}$ & $n(n-1)$ \\
\hline
\end{tabular}


TABLE 4. Spherical unipotent classes in $D_{n}, n \geq 4, n=2 m+1$, $p=2$.

\begin{tabular}{|c||c|c|c|c|}
\hline $\mathcal{O}$ & $J$ & $w(\mathcal{O})$ & $x \in \mathcal{O} \cap B w(\mathcal{O}) B$ & $\operatorname{dim} \mathcal{O}$ \\
\hline \hline $\begin{array}{c}Z_{\ell}=2^{2 \ell} \oplus 1^{2 n-4 \ell} \\
\ell=1, \ldots, m\end{array}$ & $J_{\ell}$ & $s_{\beta_{1}} s_{\delta_{1}} \cdots s_{\beta_{\ell}} s_{\delta_{\ell}}$ & $n_{\beta_{1}} n_{\delta_{1}} \cdots n_{\beta_{\ell}} n_{\delta_{\ell}}$ & $4 \ell(n-\ell)$ \\
\hline $\begin{array}{c}X_{\ell}=2_{0}^{2 \ell} \oplus 1^{2 n-4 \ell} \\
\ell=1, \ldots, m\end{array}$ & $K_{\ell}$ & $s_{\beta_{1}} \cdots s_{\beta_{\ell}}$ & $n_{\beta_{1}} \cdots n_{\beta_{\ell}}$ & $2 \ell(2 n-2 \ell-1)$ \\
\hline
\end{tabular}

We have therefore proved

Proposition 3.12. Let $\mathcal{O}$ be a non-trivial unipotent conjugacy class of $S O(2 n)$ in characteristic 2. Then $\mathcal{O}$ is spherical if and only if it consists of involutions. All non-trivial spherical unipotent classes are listed in Table 3 if $n$ is even and in Table प 4 if $n$ is odd.

Remark 3.13. From our discussion, it follows that for $D_{n}$ the map $\pi_{G}: X^{G^{\prime}} \rightarrow$ $X^{G}$ defined in [28, Theorem III.5.2] induces an isomorphism of p.o. sets between $X_{\mathrm{sph}}^{G^{\prime}}$ and $X_{\mathrm{sph}}^{G}$, where $X_{\mathrm{sph}}^{G^{\prime}}, X_{\mathrm{sph}}^{G}$ are the corresponding sets of spherical unipotent classes. In particular, every spherical unipotent conjugacy class has a representative of the form $\prod_{\alpha \in K} x_{\alpha}(1)$ for a certain set of pairwise orthogonal simple roots $K$.

3.2. Exceptional groups. For the exceptional groups, we use [21, Table 2]. In this table, for each group $G$, there are all unipotent conjugacy classes $\mathcal{O}_{u}$, in every characteristic, for which the dimension of $C(u)$ is greater than a certain number $l_{G}$. From this we deduce Table 5 where the value of $\phi C(u) / C(u)^{\circ}$ for $E_{7}$ refers to the adjoint group (see [1]).

TABLE 5. Unipotent classes of small dimension in exceptional groups.

\begin{tabular}{|c|c|c|c|c|}
\hline$G$ & $\operatorname{dim} B$ & $u$ with $\operatorname{dim} \mathcal{O}_{u} \leq \operatorname{dim} B$ & $\operatorname{dim} \mathcal{O}_{u}$ & $\varnothing C(u) / C(u)^{\circ}$ \\
\hline \hline$E_{6}$ & 42 & $A_{1}, 2 A_{1}, 3 A_{1}, A_{2}$ & $22,32,40,42$ & $1,1,1,2$ \\
\hline$E_{7}$ & 70 & $A_{1}, 2 A_{1}, 3 A_{1}^{\prime \prime}, 3 A_{1}^{\prime}, A_{2}, 4 A_{1}$ & $34,52,54,64,66,70$ & $1,1,1,1,2,1$ \\
\hline$E_{8}$ & 128 & $A_{1}, 2 A_{1}, 3 A_{1}, A_{2}, 4 A_{1}$ & $58,92,112,114,128$ & $1,1,1,2,1$ \\
\hline$F_{4}$ & 28 & $A_{1}, \tilde{A}_{1}(p=2), \tilde{A}_{1}(p \neq 2), \tilde{A}_{1}^{(2)}(p=2), A_{1} \tilde{A}_{1}$ & $16,16,22,22,28$ & $1,1,2,1,1$ \\
\hline$G_{2}$ & 8 & $A_{1}, \tilde{A}_{1}(p=3), \tilde{A}_{1}(p \neq 3), \tilde{A}_{1}^{(3)}(p=3)$ & $6,6,8,8$ & $1,1,1,1$ \\
\hline
\end{tabular}

The unipotent conjugacy classes appearing in Table 5 are the only candidates for being spherical. We shall show that they are all spherical, except for the classes of type $A_{2}$ in $E_{6}, E_{7}$ and $E_{8}$. Note that when $p=2$, then all classes of involutions appear in Table 5 by the results in [2].

Lemma 3.14. Let $\mathcal{O}$ be the unipotent conjugacy class of type $A_{2}$ in $E_{6}, E_{7}$ or $E_{8}$. Then $\mathcal{O}$ is not spherical (in any characteristic).

Proof. Let $u$ be an element in $\mathcal{O}$. From [21, Table 2], it follows that the type of $C(u)^{\circ}$ is independent of the characteristic. For completeness, we determine $C(u)^{\circ}$ in all cases.

In $E_{8}$, we may take $u=x_{\alpha_{8}}(1) x_{\beta-\alpha_{8}}(1)$, where $\beta$ is the highest root, and get $C(u)^{\circ} \leq P$, where $P$ is the maximal parabolic subgroup $P_{I \backslash\left\{\alpha_{8}\right\}}$. Then $C(u)^{\circ}=$ $C R$, where $C$ is the $E_{6}$-subgroup of $G$ corresponding to $\left\{\alpha_{1}, \ldots, \alpha_{6}\right\}$, and

$$
R=\left\{g=\prod_{\alpha \in \Phi^{+}} x_{\alpha}\left(k_{\alpha}\right) \in R_{u} P \mid k_{\alpha_{8}}=k_{\beta-\alpha_{8}}\right\} .
$$


In $E_{7}$, we may take $u=x_{\alpha_{1}}(1) x_{\beta-\alpha_{1}}(1)$, where $\beta$ is the highest root, and get $C(u)^{\circ} \leq P$, where $P$ is the maximal parabolic subgroup $P_{I \backslash\left\{\alpha_{1}\right\}}$. Then $C(u)^{\circ}=$ $C R$, where $C$ is the $A_{5}$-subgroup of $G$ corresponding to $\left\{\alpha_{2}, \alpha_{4}, \alpha_{5}, \alpha_{6}, \alpha_{7}\right\}$, and

$$
R=\left\{g=\prod_{\alpha \in \Phi^{+}} x_{\alpha}\left(k_{\alpha}\right) \in R_{u} P \mid k_{\alpha_{1}}=k_{\beta-\alpha_{1}}\right\} .
$$

In $E_{6}$, we may take $u=x_{\alpha_{2}}(1) x_{\beta-\alpha_{2}}(1)$, where $\beta$ is the highest root, and get $C(u)^{\circ} \leq P$, where $P$ is the maximal parabolic subgroup $P_{I \backslash\left\{\alpha_{2}\right\}}$. Then $C(u)^{\circ}=$ $C R$, where $C$ is the $A_{2} \times A_{2}$-subgroup of $G$ corresponding to $\left\{\alpha_{1}, \alpha_{3}, \alpha_{5}, \alpha_{6}\right\}$, and

$$
R=\left\{g=\prod_{\alpha \in \Phi^{+}} x_{\alpha}\left(k_{\alpha}\right) \in R_{u} P \mid k_{\alpha_{2}}=k_{\beta-\alpha_{2}}\right\} .
$$

It is well known that the class $A_{2}$ is not spherical in $E_{6}, E_{7}$ or $E_{8}$ over any algebraically closed field of characteristic zero ([24, Theorem (3.1)]). We may now apply [4, Theorem $2.2(\mathrm{i})$ ]. Note that the groups $C(u)^{\circ}$ involved are all defined over $\mathbb{Z}$, and the argument in the proof of [4, Theorem 2.2 (i)] is valid in our situation. Therefore $G / C(u)^{\circ}$ is not spherical in any positive characteristic. It follows that $\mathcal{O}_{u}$ is not spherical by Lemma 3.7

\subsubsection{Type $E_{6}$. We put}

$$
\begin{array}{ll}
\beta_{1}=(1,2,2,3,2,1), & \beta_{2}=(1,0,1,1,1,1), \\
\beta_{3}=(0,0,1,1,1,0), & \beta_{4}=(0,0,0,1,0,0) .
\end{array}
$$

For groups of type $E_{6}$ we have to consider $p=2$, 3. If $p=3$, then we may apply the arguments in [5, Theorem 13] to prove that the orbits of type $A_{1}, 2 A_{1}$ and $3 A_{1}$ satisfy $(*)$. Hence they are spherical, since to handle $3 A_{1}$ we need results for $D_{4}$ which hold due to [8, Theorem 3.4] and its proof (in fact what we need is that the maximal spherical unipotent conjugacy class $\mathcal{O}^{\prime}$ of $D_{4}$ satisfies $(*)$ when $p=3$ ). Therefore, now assume $p=2$. Then again we may use the proof of [5, Theorem 3.4 ] to deal with $A_{1}$ and $2 A_{1}$. Note that in these cases

$$
x_{-\beta_{1}}(1) \in A_{1} \cap B s_{\beta_{1}} B \cap U^{-}, \quad x_{-\beta_{1}}(1) x_{-\beta_{2}}(1) \in 2 A_{1} \cap B s_{\beta_{1}} s_{\beta_{2}} B \cap U^{-}
$$

with $x_{-\beta_{1}}(1) \sim n_{\beta_{1}}, x_{-\beta_{1}}(1) x_{-\beta_{2}}(1) \sim n_{\beta_{1}} n_{\beta_{2}}$. To deal with $3 A_{1}$, we may still use the arguments in the proof of [5, Theorem 3.4] since we have shown in $\S 3.1 .3$ that the maximal spherical unipotent conjugacy class $\mathcal{O}^{\prime}$ of $D_{4}$ satisfies $(*)$ when $p=2$, or directly observe that $x=n_{\beta_{1}} n_{\beta_{2}} n_{\beta_{3}} n_{\beta_{4}}$ is an involution in $B n_{\beta_{1}} n_{\beta_{2}} n_{\beta_{3}} n_{\beta_{4}} B=$ $B w_{0} B$. Then $\operatorname{dim} \mathcal{O}_{x} \geq \ell\left(w_{0}\right)+\operatorname{rk}\left(1-w_{0}\right)=40$ by Theorem 3.2. so that $x \in 3 A_{1}$ by Table 5 , since elements in $A_{2}$ are not involutions.

We have established the information presented in Tables 6 and 7 , where $w(\mathcal{O})=$ $w_{0} w_{J}$.

TABLE 6. Spherical unipotent classes in $E_{6}, p=3$ (or any $p \neq 2$ ).

\begin{tabular}{|c||c|c|c|}
\hline $\mathcal{O}$ & $J$ & $w(\mathcal{O})$ & $\operatorname{dim} \mathcal{O}$ \\
\hline \hline$A_{1}$ & $\{1,3,4,5,6\}$ & $s_{\beta_{1}}$ & 22 \\
\hline $2 A_{1}$ & $\{3,4,5\}$ & $s_{\beta_{1}} s_{\beta_{2}}$ & 32 \\
\hline $3 A_{1}$ & $\varnothing$ & $w_{0}$ & 40 \\
\hline
\end{tabular}


TABLE 7. Spherical unipotent classes in $E_{6}, p=2$.

\begin{tabular}{|c||c|c|c|c|}
\hline $\mathcal{O}$ & $J$ & $w(\mathcal{O})$ & $x \in \mathcal{O} \cap B w(\mathcal{O}) B$ & $\operatorname{dim} \mathcal{O}$ \\
\hline \hline$A_{1}$ & $\{1,3,4,5,6\}$ & $s_{\beta_{1}}$ & $n_{\beta_{1}}$ & 22 \\
\hline $2 A_{1}$ & $\{3,4,5\}$ & $s_{\beta_{1}} s_{\beta_{2}}$ & $n_{\beta_{1}} n_{\beta_{2}}$ & 32 \\
\hline $3 A_{1}$ & $\varnothing$ & $w_{0}$ & $n_{\beta_{1}} \cdots n_{\beta_{4}}$ & 40 \\
\hline
\end{tabular}

We have proved

Proposition 3.15. Let $\mathcal{O}$ be a non-trivial unipotent conjugacy class of $E_{6}$. Then $\mathcal{O}$ is spherical if and only if it is listed in Tables 6 or 7. If $p=2$, these are precisely the classes consisting of involutions.

We observe that, independent of the characteristic, $x_{\alpha_{1}}(1) \in A_{1}, x_{\alpha_{1}}(1) x_{\alpha_{4}}(1) \in$ $2 A_{1}$ and $x_{\alpha_{1}}(1) x_{\alpha_{4}}(1) x_{\alpha_{6}}(1) \in 3 A_{1}$. Hence every spherical unipotent conjugacy class has a representative of the form $\prod_{\alpha \in K} x_{\alpha}(1)$ for a certain set of pairwise orthogonal simple roots $K$.

3.2.2. Type $E_{7}$. We put

$$
\begin{aligned}
& \beta_{1}=(2,2,3,4,3,2,1), \quad \beta_{2}=(0,1,1,2,2,2,1), \beta_{3}=(0,1,1,2,1,0,0), \\
& \beta_{4}=\alpha_{7}, \quad \beta_{5}=\alpha_{5}, \quad \beta_{6}=\alpha_{3}, \quad \beta_{7}=\alpha_{2} .
\end{aligned}
$$

For groups of type $E_{7}$ we have to consider $p=2,3$. If $p=3$, then we may apply the arguments in [5. Theorem 13] to prove that the orbits of type $A_{1}, 2 A_{1},\left(3 A_{1}\right)^{\prime}$, $\left(3 A_{1}\right)^{\prime \prime}$ and $4 A_{1}$ are spherical, since we need results for $D_{n}$ which hold due to [8, Theorem 3.4] and its proof. So now assume $p=2$. Then again we may use the proof of [5, Theorem 3.4] to deal with $A_{1}, 2 A_{1}$ and $\left(3 A_{1}\right)^{\prime \prime}$. Note that in these cases

$$
\begin{gathered}
x_{-\beta_{1}}(1) \in A_{1} \cap B s_{\beta_{1}} B \cap U^{-}, \quad x_{-\beta_{1}}(1) x_{-\beta_{2}}(1) \in 2 A_{1} \cap B s_{\beta_{1}} s_{\beta_{2}} B \cap U^{-}, \\
x_{-\beta_{1}}(1) x_{-\beta_{2}}(1) x_{-\beta_{4}}(1) \in\left(3 A_{1}\right)^{\prime \prime} \cap B s_{\beta_{1}} s_{\beta_{2}} s_{\beta_{4}} B \cap U^{-} .
\end{gathered}
$$

To deal with $\left(3 A_{1}\right)^{\prime}$ and $4 A_{1}$, again we may apply the arguments in 5 , Theorem 13], since we need results for $D_{n}$ when $p=2$, which we proved in $\S 3.1 .3$. However, it is also possible to show directly that $n_{\beta_{1}} n_{\beta_{2}} n_{\beta_{3}} n_{\beta_{6}} \in s_{\beta_{1}} s_{\beta_{2}} s_{\beta_{3}} s_{\beta_{6}} B \cap\left(3 A_{1}\right)^{\prime}$. To deal with $4 A_{1}$ one can observe that $x=n_{\beta_{1}} \cdots n_{\beta_{7}}$ is an involution in $B w_{0} B$. Then $\operatorname{dim} \mathcal{O}_{x} \geq \ell\left(w_{0}\right)+\operatorname{rk}\left(1-w_{0}\right)=70$ by Theorem 3.2 so that $x \in 4 A_{1}$ by Table 5 ,

We have established the information presented in Tables 8 and 9 , where $w(\mathcal{O})=$ $w_{0} w_{J}$.

TABLE 8. Spherical unipotent classes in $E_{7}, p=3$ (or any $p \neq 2$ ).

\begin{tabular}{|c||c|c|c|}
\hline $\mathcal{O}$ & $J$ & $w(\mathcal{O})$ & $\operatorname{dim} \mathcal{O}$ \\
\hline \hline$A_{1}$ & $\{2,3,4,5,6,7\}$ & $s_{\beta_{1}}$ & 34 \\
\hline $2 A_{1}$ & $\{2,3,4,5,7\}$ & $s_{\beta_{1}} s_{\beta_{2}}$ & 52 \\
\hline$\left(3 A_{1}\right)^{\prime \prime}$ & $\{2,3,4,5\}$ & $s_{\beta_{1}} s_{\beta_{2}} s_{\beta_{4}}$ & 54 \\
\hline$\left(3 A_{1}\right)^{\prime}$ & $\{2,5,7\}$ & $s_{\beta_{1}} s_{\beta_{2}} s_{\beta_{3}} s_{\beta_{6}}$ & 64 \\
\hline $4 A_{1}$ & $\varnothing$ & $w_{0}$ & 70 \\
\hline
\end{tabular}


TABLE 9. Spherical unipotent classes in $E_{7}, p=2$.

\begin{tabular}{|c||c|c|c|c|}
\hline $\mathcal{O}$ & $J$ & $w(\mathcal{O})$ & $x \in \mathcal{O} \cap B w(\mathcal{O}) B$ & $\operatorname{dim} \mathcal{O}$ \\
\hline \hline$A_{1}$ & $\{2,3,4,5,6,7\}$ & $s_{\beta_{1}}$ & $n_{\beta_{1}}$ & 34 \\
\hline $2 A_{1}$ & $\{2,3,4,5,7\}$ & $s_{\beta_{1}} s_{\beta_{2}}$ & $n_{\beta_{1}} n_{\beta_{2}}$ & 52 \\
\hline$\left(3 A_{1}\right)^{\prime \prime}$ & $\{2,3,4,5\}$ & $s_{\beta_{1}} s_{\beta_{2}} s_{\beta_{4}}$ & $n_{\beta_{1}} n_{\beta_{2}} n_{\beta_{4}}$ & 54 \\
\hline$\left(3 A_{1}\right)^{\prime}$ & $\{2,5,7\}$ & $s_{\beta_{1}} s_{\beta_{2}} s_{\beta_{3}} s_{\beta_{6}}$ & $n_{\beta_{1}} n_{\beta_{2}} n_{\beta_{3}} n_{\beta_{6}}$ & 64 \\
\hline $4 A_{1}$ & $\varnothing$ & $w_{0}$ & $n_{\beta_{1}} \cdots n_{\beta_{7}}$ & 70 \\
\hline
\end{tabular}

We have proved

Proposition 3.16. Let $\mathcal{O}$ be a non-trivial unipotent conjugacy class of $E_{7}$. Then $\mathcal{O}$ is spherical if and only if it is listed in Table $\mathbf{8}$ or 9 . If $p=2$, these are precisely the classes consisting of involutions.

We observe that, independent of the characteristic, $x_{\alpha_{1}}(1) \in A_{1}, x_{\alpha_{1}}(1) x_{\alpha_{4}}(1)$ $\in 2 A_{1}, \quad x_{\alpha_{3}}(1) x_{\alpha_{5}}(1) x_{\alpha_{7}}(1) \in\left(3 A_{1}\right)^{\prime \prime}, \quad x_{\alpha_{1}}(1) x_{\alpha_{4}}(1) x_{\alpha_{6}}(1) \in\left(3 A_{1}\right)^{\prime}$ and $x_{\alpha_{2}}(1) x_{\alpha_{3}}(1) x_{\alpha_{5}}(1) x_{\alpha_{7}}(1) \in 4 A_{1}$. Hence every spherical unipotent conjugacy class has a representative of the form $\prod_{\alpha \in K} x_{\alpha}(1)$ for a certain set of pairwise orthogonal simple roots $K$.

\subsubsection{Type $E_{8}$. We put}

$$
\begin{aligned}
& \beta_{1}=(2,3,4,6,5,4,3,2), \beta_{2}=(2,2,3,4,3,2,1,0), \beta_{3}=(0,1,1,2,2,2,1,0), \\
& \beta_{4}=(0,1,1,2,1,0,0,0), \beta_{5}=\alpha_{7}, \beta_{6}=\alpha_{5}, \beta_{7}=\alpha_{3}, \beta_{8}=\alpha_{2} .
\end{aligned}
$$

For groups of type $E_{8}$ we have to consider $p=2,3,5$. If $p=3$ or 5 , then we may apply the arguments in [5. Theorem 13] to prove that the orbits of types, $A_{1}, 2 A_{1}$, $3 A_{1}$ and $4 A_{1}$ are spherical, since to handle $3 A_{1}$ and $4 A_{1}$ we need results for $D_{4}$ and $D_{6}$ which hold due to [ 8 , Theorem 3.4] and its proof. Therefore, now assume $p=2$. Then again we may use the proof of [5, Theorem 3.4] to deal with $A_{1}$ and $2 A_{1}$. Note that in these cases

$$
\begin{gathered}
x_{-\beta_{1}}(1) \in A_{1} \cap B s_{\beta_{1}} B \cap U^{-}, \\
x_{-\beta_{1}}(1) x_{-\beta_{2}}(1) \in 2 A_{1} \cap B s_{\beta_{1}} s_{\beta_{2}} B \cap U^{-} .
\end{gathered}
$$

To deal with $3 A_{1}$ and $4 A_{1}$, again we may apply the arguments in [5, Theorem 13], since we need results for $D_{n}$ when $p=2$, which we proved in $\S 3.1 .3$. However, it is also possible to show directly that $n_{\beta_{1}} n_{\beta_{2}} n_{\beta_{3}} n_{\beta_{5}} \in s_{\beta_{1}} s_{\beta_{2}} s_{\beta_{3}} s_{\beta_{5}} B \cap 3 A_{1}$. To deal with $4 A_{1}$ one can observe that $x=n_{\beta_{1}} \cdots n_{\beta_{8}}$ is an involution in $B w_{0} B$. Then $\operatorname{dim} \mathcal{O}_{x} \geq \ell\left(w_{0}\right)+\operatorname{rk}\left(1-w_{0}\right)=128$ by Theorem 3.2, so that $x \in 4 A_{1}$ by Table 5 .

We have established the information presented in Tables [10 and [1], where $w(\mathcal{O})=w_{0} w_{J}$.

We have proved

Proposition 3.17. Let $\mathcal{O}$ be a non-trivial unipotent conjugacy class of $E_{8}$. Then $\mathcal{O}$ is spherical if and only if it is listed in Table 10 or 11, If $p=2$, these are precisely the classes consisting of involutions.

We observe that, independent of the characteristic, $x_{\alpha_{1}}(1) \in A_{1}, x_{\alpha_{1}}(1) x_{\alpha_{4}}(1) \in$ $2 A_{1}, x_{\alpha_{1}}(1) x_{\alpha_{4}}(1) x_{\alpha_{6}}(1) \in 3 A_{1}$, and $x_{\alpha_{1}}(1) x_{\alpha_{4}}(1) x_{\alpha_{6}}(1) x_{\alpha_{8}}(1) \in 4 A_{1}$. Hence every spherical unipotent conjugacy class has a representative of the form $\prod_{\alpha \in K} x_{\alpha}(1)$ for a certain set of pairwise orthogonal simple roots $K$. 
TABLE 10. Spherical unipotent classes in $E_{8}, p=3$ or 5 (or any $p \neq 2$ ).

\begin{tabular}{|c||c|c|c|}
\hline $\mathcal{O}$ & $J$ & $w(\mathcal{O})$ & $\operatorname{dim} \mathcal{O}$ \\
\hline \hline$A_{1}$ & $\{1,2,3,4,5,6,7\}$ & $s_{\beta_{1}}$ & 58 \\
\hline $2 A_{1}$ & $\{2,3,4,5,6,7\}$ & $s_{\beta_{1}} s_{\beta_{2}}$ & 92 \\
\hline $3 A_{1}$ & $\{2,3,4,5\}$ & $s_{\beta_{1}} s_{\beta_{2}} s_{\beta_{3}} s_{\beta_{5}}$ & 112 \\
\hline $4 A_{1}$ & $\varnothing$ & $w_{0}$ & 128 \\
\hline
\end{tabular}

TABLE 11. Spherical unipotent classes in $E_{8}, p=2$.

\begin{tabular}{|c||c|c|c|c|}
\hline $\mathcal{O}$ & $J$ & $w(\mathcal{O})$ & $x \in \mathcal{O} \cap B w(\mathcal{O}) B$ & $\operatorname{dim} \mathcal{O}$ \\
\hline \hline$A_{1}$ & $\{1,2,3,4,5,6,7\}$ & $s_{\beta_{1}}$ & $n_{\beta_{1}}$ & 58 \\
\hline $2 A_{1}$ & $\{2,3,4,5,6,7\}$ & $s_{\beta_{1}} s_{\beta_{2}}$ & $n_{\beta_{1}} n_{\beta_{2}}$ & 92 \\
\hline $3 A_{1}$ & $\{2,3,4,5\}$ & $s_{\beta_{1}} s_{\beta_{2}} s_{\beta_{3}} s_{\beta_{5}}$ & $n_{\beta_{1}} n_{\beta_{2}} n_{\beta_{3}} n_{\beta_{5}}$ & 112 \\
\hline $4 A_{1}$ & $\varnothing$ & $w_{0}$ & $n_{\beta_{1}} \cdots n_{\beta_{8}}$ & 128 \\
\hline
\end{tabular}

3.2.4. Type $F_{4}$. We put

$$
\begin{aligned}
& \beta_{1}=(2,3,4,2), \quad \beta_{2}=(0,1,2,2), \\
& \beta_{3}=(0,1,2,0), \quad \beta_{4}=(0,1,0,0) ;
\end{aligned}
$$

also $\gamma_{1}$ is the highest short root $(1,2,3,2)$.

For groups of type $F_{4}$ we have to consider $p=2$, 3. If $p=3$, then we may apply the arguments in [5. Theorem 13] to prove that the orbits of types $A_{1}, \tilde{A}_{1}$ and $A_{1} \tilde{A}_{1}$ are spherical, since to handle $A_{1} \tilde{A}_{1}$ we need results for $D_{4}$ which hold due to [8, Theorem 3.4] and its proof. Therefore, now assume $p=2$. Here there are more conjugacy classes $\mathcal{O}_{u}$ (due to the presence of the graph automorphism of $G$ ) for which $\operatorname{dim} \mathcal{O}_{u} \leq \operatorname{dim} B$ (see Table 5). Each class consists of involutions. We may take the following representatives:

$$
\begin{aligned}
& x_{-\beta_{1}}(1) \in A_{1} \cap B s_{\beta_{1}} B \cap U^{-}, \\
& x_{-\gamma_{1}}(1) \in \tilde{A}_{1} \cap B s_{\gamma_{1}} B \cap U^{-} .
\end{aligned}
$$

To deal with $\tilde{A}_{1}^{(2)}$, we observe that $u=x_{\beta_{1}}(1) x_{\gamma_{1}}(1) \in \tilde{A}_{1}^{(2)}$ by [2, (13.1)]. Let $K$ be the $C_{2}$-subgroup of $G$ with basis $\left\{(1,1,1,0), \beta_{2}\right\}$. Then $\beta_{1}$ and $\gamma_{1}$ are the highest long and short roots in $K$, respectively. A direct calculation in $C_{2}$ shows that $u$ is conjugate to $v=x_{\beta_{1}}(1) x_{\beta_{2}}(1)$; hence

$$
x_{-\beta_{1}}(1) x_{-\beta_{2}}(1) \in \tilde{A}_{1}^{(2)} \cap B s_{\beta_{1}} s_{\beta_{2}} B \cap U^{-} .
$$

Finally, to deal with $A_{1} \tilde{A}_{1}$ we observe that $x=n_{\beta_{1}} \cdots n_{\beta_{4}}$ is an involution in $B w_{0} B$. Then $\operatorname{dim} \mathcal{O}_{x} \geq \ell\left(w_{0}\right)+\operatorname{rk}\left(1-w_{0}\right)=28$ by Theorem 3.2 so that $x \in A_{1} \tilde{A}_{1}$ by Table 5. We have established the information presented in Tables 12 and 13, where $w(\mathcal{O})=w_{0} w_{J}$.

Proposition 3.18. Let $\mathcal{O}$ be a non-trivial unipotent conjugacy class in $F_{4}$. Then $\mathcal{O}$ is spherical if and only if it is listed in Table 12 or 13. If $p=2$, these are precisely the classes consisting of involutions.

We note that in $G_{\mathbb{C}}$ there is an involution $\sigma$ such that $C(\sigma)$ is of type $B_{4}$ and such that $\mathcal{O}_{\sigma}$ lies over $s_{\gamma_{1}}$. We also observe that if $p=2$, then $x_{\alpha_{4}}(1) \in A_{1}$, 
TABLE 12. Spherical unipotent classes in $F_{4}, p=3$ (or any $p \neq 2$ ).

\begin{tabular}{|c||c|c|c|}
\hline $\mathcal{O}$ & $J$ & $w(\mathcal{O})$ & $\operatorname{dim} \mathcal{O}$ \\
\hline \hline$A_{1}$ & $\{2,3,4\}$ & $s_{\beta_{1}}$ & 16 \\
\hline$\tilde{A}_{1}$ & $\{2,3\}$ & $s_{\beta_{1}} s_{\beta_{2}}$ & 22 \\
\hline$A_{1} \tilde{A}_{1}$ & $\emptyset$ & $w_{0}$ & 28 \\
\hline
\end{tabular}

TABLE 13. Spherical unipotent classes in $F_{4}, p=2$.

\begin{tabular}{|c||c|c|c|c|}
\hline $\mathcal{O}$ & $J$ & $w(\mathcal{O})$ & $x \in \mathcal{O} \cap B w(\mathcal{O}) B$ & $\operatorname{dim} \mathcal{O}$ \\
\hline \hline$A_{1}$ & $\{2,3,4\}$ & $s_{\beta_{1}}$ & $n_{\beta_{1}}$ & 16 \\
\hline$\tilde{A}_{1}$ & $\{1,2,3\}$ & $s_{\gamma_{1}}$ & $n_{\gamma_{1}}$ & 16 \\
\hline$\tilde{A}_{1}^{(2)}$ & $\{2,3\}$ & $s_{\beta_{1}} s_{\beta_{2}}$ & $n_{\beta_{1}} n_{\beta_{2}}$ & 22 \\
\hline$A_{1} \tilde{A}_{1}$ & $\emptyset$ & $w_{0}$ & $n_{\beta_{1}} n_{\beta_{2}} n_{\beta_{3}} n_{\beta_{4}}$ & 28 \\
\hline
\end{tabular}

$x_{\alpha_{1}}(1) \in \tilde{A}_{1}, x_{\alpha_{1}}(1) x_{\alpha_{3}}(1) \in A_{1} \tilde{A}_{1}$ and these exhaust the conjugacy classes of involutions with a representative of the form $\prod_{i \in K} x_{\alpha_{i}}(1), K \subseteq \Pi$. In particular, $\tilde{A}_{1}^{(2)}$ has no representative of the form $\prod_{i \in K} x_{\alpha_{i}}(1)$.

3.2.5. Type $G_{2}$. We put $\beta_{1}=(3,2), \beta_{2}=\alpha_{1}, \gamma_{1}=(2,1)$ (the highest short root).

For groups of type $G_{2}$ we have to consider $p=2,3$. The p.o. set of unipotent conjugacy classes is described in the tables in [28, Proposition II 10.4].

If $p=2$, the classification of unipotent conjugacy classes $\mathcal{O}$ for which $\operatorname{dim} \mathcal{O} \leq$ $\operatorname{dim} B$ is the same as over $\mathbb{C}$ and each class consists of involutions. We may take

$$
x_{-\beta_{1}}(1) \in A_{1} \cap B s_{\beta_{1}} B \cap U^{-} .
$$

To deal with $\tilde{A}_{1}$ we observe that $x=n_{\beta_{1}} n_{\beta_{2}}$ is an involution in $B w_{0} B$. Then $\operatorname{dim} \mathcal{O}_{x} \geq \ell\left(w_{0}\right)+\operatorname{rk}\left(1-w_{0}\right)=8$ by Theorem 3.2. so that $x \in \tilde{A}_{1}$ by Table 5 .

Therefore, now assume $p=3$. Here there are more conjugacy classes $\mathcal{O}$ for which $\operatorname{dim} \mathcal{O}_{u} \leq \operatorname{dim} B$ (see Table 5), due to the presence of the graph automorphism of $G$. We may take the following representatives:

$$
\begin{aligned}
& x_{-\beta_{1}}(1) \in A_{1} \cap B s_{\beta_{1}} B \cap U^{-}, \\
& x_{-\gamma_{1}}(1) \in \tilde{A}_{1} \cap B s_{\gamma_{1}} B \cap U^{-} .
\end{aligned}
$$

To deal with $\tilde{A}_{1}^{(3)}$, we observe that since $A_{1} \leq \tilde{A}_{1}^{(3)}$ and $\tilde{A}_{1} \leq \tilde{A}_{1}^{(3)}$, we get $s_{\beta_{1}} \leq$ $w\left(\tilde{A}_{1}^{(3)}\right)$ and $s_{\gamma_{1}} \leq w\left(\tilde{A}_{1}^{(3)}\right)$ so that $w\left(\tilde{A}_{1}^{(3)}\right)=w_{0}$, and we are done since $\operatorname{dim} \tilde{A}_{1}^{(3)}=$ 8.

We have established the information presented in Tables 14 and 15, where $w(\mathcal{O})=w_{0} w_{J}$.

TABlE 14. Spherical unipotent classes in $G_{2}, p=2$.

\begin{tabular}{|c||c|c|c|c|}
\hline $\mathcal{O}$ & $J$ & $w(\mathcal{O})$ & $x \in \mathcal{O} \cap B w(\mathcal{O}) B$ & $\operatorname{dim} \mathcal{O}$ \\
\hline \hline$A_{1}$ & $\{1\}$ & $s_{\beta_{1}}$ & $n_{\beta_{1}}$ & 6 \\
\hline$\tilde{A}_{1}$ & $\emptyset$ & $s_{\beta_{1}} s_{\beta_{2}}$ & $n_{\beta_{1}} n_{\beta_{2}}$ & 8 \\
\hline
\end{tabular}


TABLE 15. Spherical unipotent classes in $G_{2}, p=3$.

\begin{tabular}{|c||c|c|c|}
\hline $\mathcal{O}$ & $J$ & $w(\mathcal{O})$ & $\operatorname{dim} \mathcal{O}$ \\
\hline \hline$A_{1}$ & $\{1\}$ & $s_{\beta_{1}}$ & 6 \\
\hline$\tilde{A}_{1}$ & $\{2\}$ & $s_{\gamma_{1}}$ & 6 \\
\hline$\tilde{A}_{1}^{(3)}$ & $\emptyset$ & $s_{\beta_{1}} s_{\beta_{2}}$ & 8 \\
\hline
\end{tabular}

Proposition 3.19. Let $\mathcal{O}$ be a non-trivial unipotent conjugacy class in $G_{2}$. Then $\mathcal{O}$ is spherical if and only if it is listed in Table 14 or 15. If $p=2$, these are precisely the classes consisting of involutions.

Note that if $p=3$, then $x_{\alpha_{2}}(1) \in A_{1}, x_{\alpha_{1}}(1) \in \tilde{A}_{1}$, while $\tilde{A}_{1}^{(3)}$ has no representative of the form $\prod_{i \in K} x_{\alpha_{i}}(1), K \subseteq \Pi$.

This completes the classification of unipotent spherical conjugacy classes in bad characteristic and the proof of Theorem 3.4, which clearly holds for every connected reductive algebraic group in characteristic 2 .

\section{Symmetric homogeneous SPaCes}

In this section we shall prove that if $G$ is a connected reductive algebraic group over an algebraically closed field $k$ of characteristic 2 , then $H=C(\sigma)$ is a spherical subgroup of $G$ for every involutory automorphism $\sigma$ of $G$. This was proved by Vust in [32] in characteristic zero (see also [23] over $\mathbb{C}$ ). Then Springer extended the result to odd characteristic in 29]. In [27, Seitz gives an alternative proof of Springer's result.

We start by observing that it is enough to assume $G$ simple, for if $G$ is connected reductive, then $G=S G_{1} \cdots G_{t}$, where $S$ is the connected component of $Z(G)$ and $G_{1}, \ldots, G_{t}$ are the simple components of $G$. Let $\sigma$ be an involutory automorphism of $G$. Then $\sigma$ fixes $S$ and induces a permutation $\rho$ of the set $\{1, \ldots, t\}$. If $\rho$ is non-trivial, then it is the product of disjoint cycles of length 2. Suppose one of these cycles is $(1,2)$. Then $\sigma$ induces an isomorphism $\varphi: G_{1} \rightarrow G_{2}$. Let $B_{1}=T_{1} U_{1}$ be a Borel subgroup of $G_{1}$, where $U_{1}$ is the unipotent radical of $B_{1}$ and $T_{1}$ is a maximal torus. Let $V_{1}$ be the maximal unipotent subgroup of the Borel subgroup of $G_{1}$ opposite to $B_{1}$, and let $R=B_{1} V_{1}^{\varphi} \leq G_{1} G_{2}$. Then $C_{G_{1} G_{2}}(\sigma) \cap R$ is finite so that $C_{G_{1} G_{2}}(\sigma)$ is a spherical subgroup of $G_{1} G_{2}$, since $\operatorname{dim} C_{G_{1} G_{2}}(\sigma)+\operatorname{dim} B_{1} V_{1}^{\varphi}=$ $\operatorname{dim} G_{1} G_{2}$. Of course $C_{S}(\sigma)$ is a spherical subgroup of $S$, so to conclude it is enough to assume that $G$ is a (connected) simple algebraic group.

In the following we assume that $G$ is a simple algebraic group over $k$, of any characteristic. We shall use a generalization of Theorem 3.2.

Let $\tau$ be an automorphism of $G$ fixing $B$ and $T$, and consider $G:\langle\tau\rangle$. Assume $\tau$ has order $r$. Then we have the Bruhat decomposition

$$
G:\langle\tau\rangle=\bigcup_{w \in W, i \in \mathbb{Z}_{r}} B \tau^{i} w B
$$

Let $\mathcal{O}$ be a $G$-orbit in $G:\langle\tau\rangle$. Then there exists a unique $i \in \mathbb{Z}_{r}$ such that $\mathcal{O} \subseteq \bigcup_{w \in W} B \tau^{i} w B$, and there is a unique $z=z(\mathcal{O})$ such that $\mathcal{O} \cap B \tau^{i} z B$ is open dense in $\mathcal{O}$. In particular,

$$
\overline{\mathcal{O}}=\overline{\mathcal{O} \cap B \tau^{i} z B} \subseteq \overline{B \tau^{i} z B}=\tau^{i} \overline{B z B} .
$$


It follows that if $y$ is an element of $\overline{\mathcal{O}}$ and $y \in B \tau^{i} w B$, then $w \leq z$ in the Chevalley-Bruhat order of $W$. Let us observe that if $\mathcal{O}$ is spherical and B.x is the dense $B$-orbit in $\mathcal{O}$, then $B . x \subseteq B \tau^{i} z B$.

We still denote by $\tau$ the automorphism of $E=X(T) \otimes \mathbb{R}$ induced by $\tau$ (i.e. $\tau(\chi)(t)=\chi\left(\tau^{-1} t \tau\right)$ for every $\left.\chi \in X=X(T), t \in T\right)$. For every $w \in W$ we put

$$
T^{\tau w}=\left\{t \in T \mid w^{-1} \tau^{-1} t \tau w=t\right\} .
$$

We have $\operatorname{dim} T^{\tau w}=n-\operatorname{rk}(1-\tau w)$.

Theorem 4.1. Let $\sigma \in G:\langle\tau\rangle, \sigma=\tau^{i} g$, for a certain $g \in G, i \in \mathbb{Z}_{r}$, and let $\mathcal{O}=G . \sigma$. Suppose that $\mathcal{O}$ contains an element $x \in B \tau^{i} w B$, for a certain $w \in W$, where $U_{w^{-1}}$ is $\tau$-invariant. Then

$$
\operatorname{dim} B \cdot x \geq \ell(w)+\operatorname{rk}\left(1-\tau^{i} w\right) .
$$

In particular, $\operatorname{dim} \mathcal{O} \geq \ell(w)+\operatorname{rk}\left(1-\tau^{i} w\right)$. If, in addition,

$$
\operatorname{dim} \mathcal{O} \leq \ell(w)+\operatorname{rk}\left(1-\tau^{i} w\right),
$$

then $\mathcal{O}$ is spherical, $w=z(\mathcal{O})$ and B.x is the dense B-orbit in $\mathcal{O}$.

Proof. Without loss of generality, we may assume $x=\tau^{i} \dot{w} u$, for a certain representative $\dot{w}$ of $w$ in $N$ and $u \in U$. Let us estimate the dimension of the orbit $B_{w^{-1} . x}$, where $B_{w^{-1}}=T U_{w^{-1}}$.

Let $v t \in C_{B_{w^{-1}}}(x)$, with $v \in U_{w^{-1}}, t \in T$. Then

$$
\tau^{i} \dot{w} u v t=v t \tau^{i} \dot{w} u=\tau^{i} \tau^{-i} v t \tau^{i} \dot{w} u=\tau^{i} \tau^{-i} v \tau^{i} \tau^{-i} t \tau^{i} \dot{w} u=\tau^{i} \tau^{-i} v \tau^{i} \dot{w} \dot{w}^{-1} \tau^{-i} t \tau^{i} \dot{w} u
$$

so that, by the uniqueness of the decomposition, $v=1$ since $\tau^{-i} v \tau^{i} \in U_{w^{-1}}$. Moreover, from $u t=\dot{w}^{-1} \tau^{-i} t \tau^{i} \dot{w} u$ it follows that $t=\dot{w}^{-1} \tau^{-i} t \tau^{i} \dot{w}$. Therefore $C_{B_{w^{-1}}}(x) \leq T^{\tau^{i} w}$. Thus $\operatorname{dim} C_{B_{w^{-1}}}(x) \leq \operatorname{dim} T^{\tau^{i} w}=n-\operatorname{rk}\left(1-\tau^{i} w\right)$ and

$$
\begin{aligned}
\operatorname{dim} B_{w^{-1}} . x & =\operatorname{dim} B_{w^{-1}}-\operatorname{dim} C_{B_{w^{-1}}}(x) \geq \ell(w)+n-n+\operatorname{rk}\left(1-\tau^{i} w\right) \\
& =\ell(w)+\operatorname{rk}\left(1-\tau^{i} w\right) .
\end{aligned}
$$

If, in addition, $\ell(w)+\operatorname{rk}\left(1-\tau^{i} w\right) \geq \operatorname{dim} \mathcal{O}$, then $\operatorname{dim} \mathcal{O}=\ell(w)+\operatorname{rk}\left(1-\tau^{i} w\right)$. In particular B.x is the dense $B$-orbit in $\mathcal{O}$.

We observe that the condition $\tau\left(U_{w^{-1}}\right)=U_{w^{-1}}$ is clearly satisfied if $w=$ $s_{r_{1}} \cdots s_{r_{k}}$, where $r_{1}, \ldots, r_{k}$ are roots fixed by $\tau$, or if $\left\{r_{1}, \ldots, r_{k}\right\}$ is a $\tau$-invariant set of pairwise orthogonal roots.

In the remainder of this section we assume that the characteristic of $k$ is 2 .

In the previous section we have already shown that $C(\sigma)$ is a spherical subgroup of $G$ when $\sigma$ is an inner involution of $G$. We are therefore left to deal with outer involutions, which exist only in the following cases: $A_{\ell}, \ell \geq 2, D_{\ell}, \ell \geq 4$ and $E_{6}$.

To prove that the fixed point subgroup of any outer involution of $G$ is spherical, we shall use the classification of outer involutions of $G$ as in [28] and [2]. We fix the graph automorphism $\tau$ (of order 2) of $G$, and for each $G$-orbit $\mathcal{O}$ of outer involutions of $G$ we show that there exists an involution $w=s_{\delta_{1}} \cdots s_{\delta_{\ell}} \in W$ such that $\mathcal{O} \cap B \tau w B$ is not empty, $\operatorname{dim} \mathcal{O}=\ell(w)+\operatorname{rk}(1-\tau w)$, the $\delta_{i}$ 's are pairwise orthogonal positive roots and $\left\{\delta_{1}, \ldots, \delta_{\ell}\right\}$ is $\tau$-invariant. By Theorem 4.1, $\mathcal{O}$ is spherical (with $z(\mathcal{O})=w$ ). We consider the various cases: if $\sigma=\tau g \in G:\langle\tau\rangle$, $C(\sigma)$ stands for $C_{G}(\sigma)$. In each case we use the notation introduced in Section 3 . 
4.1. Type $A_{n}, n=2 m, m \geq 1$. We take $G=S L(2 m+1)$. In this case there is only one (class of) outer involution $\tau$, the graph automorphism of $S L(2 m+1)$, and $C(\tau)=S O(2 m+1)$. Then

$$
\operatorname{dim} S L(2 m+1) / S O(2 m+1)=2 m^{2}+3 m,
$$

which is the dimension of a Borel subgroup of $S L(2 m+1)$. We may take

$$
x=\tau n_{\beta_{1}} \cdots n_{\beta_{m}} \in \tau w_{0} B .
$$

Then $x^{2}=1$ since $\tau\left(\beta_{k}\right)=\beta_{k}$ for each $k$, so that $x$ lies in $\mathcal{O}_{\tau}$. Since $\tau w_{0}=-1$, we get

$$
\ell\left(w_{0}\right)+\operatorname{rk}\left(1-\tau w_{0}\right)=\operatorname{dim} B,
$$

and we are done.

We have established the information presented in Table 16, where $w(\mathcal{O})=w_{0} w_{J}$.

TABLE 16. Outer involutions of $S L(2 m+1), m \geq 1, p=2$.

\begin{tabular}{|c||c|c|c|c|}
\hline $\mathcal{O}$ & $J$ & $w(\mathcal{O})$ & $x \in \mathcal{O} \cap \tau B w(\mathcal{O}) B$ & $\operatorname{dim} \mathcal{O}$ \\
\hline \hline$\tau$ & $\emptyset$ & $w_{0}=s_{\beta_{1}} \cdots s_{\beta_{m}}$ & $\tau n_{\beta_{1}} \cdots n_{\beta_{m}}$ & $2 m^{2}+3 m$ \\
\hline
\end{tabular}

4.2. Type $A_{n}, n=2 m-1, m \geq 2$. We take $G=S L(2 m)$. In this case there are two (classes of) outer involutions: $\tau$ and $\tau x_{\beta_{1}}(1)$, with $C(\tau)=S p(2 m)$, $C\left(\tau x_{\beta_{1}}(1)\right)=C_{S p(2 m)}\left(x_{\beta_{1}}(1)\right)$. We have

$$
\operatorname{dim} S L(2 m) / S p(2 m)=2 m^{2}-m-1 .
$$

We put $J=\{1,3, \ldots, n\}, w=w_{0} w_{J}$. We have

$$
\ell(w)=\ell\left(w_{0}\right)-\ell\left(w_{J}\right)=m(2 m-1)-m=2 m^{2}-2 m
$$

and

$$
\operatorname{rk}(1-\tau w)=\operatorname{rk}\left(w_{J}+1\right)=m-1
$$

since $\tau w_{0}=-1$ and the (simple) roots in $J$ are pairwise orthogonal. Hence

$$
\ell(w)+\operatorname{rk}(1-\tau w)=2 m^{2}-m-1=\operatorname{dim} S L(2 m) / S p(2 m) .
$$

We are left to exhibit a conjugate $x \in \tau B w B$ of $\tau$. For this purpose we distinguish 2 cases.

Assume $m$ is even, $m=2 r$. Then there are precisely $m$ positive roots $\gamma_{1}, \ldots, \gamma_{m}$ for which $w(\gamma)=-\gamma$, namely

$$
\gamma_{2 i-1}=e_{2 i-1}-e_{2 m-2 i+1}, \quad \gamma_{2 i}=e_{2 i}-e_{2 m+2-2 i}
$$

for $i=1, \ldots, r$ and

$$
w=s_{\gamma_{1}} \cdots s_{\gamma_{m}}
$$

We also note that $\tau$ exchanges $\gamma_{2 i-1}$ and $\gamma_{2 i}$ for each $i=1, \ldots, r$. In this case we take

$$
x=g \tau g^{-1},
$$

where $g$ is the involution $g=x_{-\gamma_{1}}(1) x_{-\gamma_{3}}(1) \cdots x_{-\gamma_{m-1}}(1)$. Then

$$
x=\tau x_{-\gamma_{1}}(1) x_{-\gamma_{2}}(1) x_{-\gamma_{3}}(1) \cdots x_{-\gamma_{m-1}}(1) x_{-\gamma_{m}}(1) \in \tau B w B,
$$

and we are done. 
If $m$ is odd, $m-1=2 r$, then there are precisely $m-1$ positive roots $\gamma_{1}, \ldots, \gamma_{m-1}$ for which $w(\gamma)=-\gamma$, namely

$$
\gamma_{2 i-1}=e_{2 i-1}-e_{2 m-2 i+1}, \quad \gamma_{2 i}=e_{2 i}-e_{2 m+2-2 i}
$$

for $i=1, \ldots, r$ and

$$
w=s_{\gamma_{1}} \cdots s_{\gamma_{m-1}} .
$$

We also note that $\tau$ exchanges $\gamma_{2 i-1}$ and $\gamma_{2 i}$ for each $i=1, \ldots, r$. In this case we take

$$
x=g \tau g^{-1},
$$

where $g$ is the involution $g=x_{-\gamma_{1}}(1) x_{-\gamma_{3}}(1) \cdots x_{-\gamma_{m-2}}(1)$. Then

$$
x=\tau x_{-\gamma_{1}}(1) x_{-\gamma_{2}}(1) x_{-\gamma_{3}}(1) \cdots x_{-\gamma_{m-2}}(1) x_{-\gamma_{m-1}}(1) \in \tau B w B,
$$

and we are done.

We finally deal with $\tau x_{\beta_{1}}(1), H=C\left(\tau x_{\beta_{1}}(1)\right)=C_{S p(2 m)}\left(x_{\beta_{1}}(1)\right)$. We have

$\operatorname{dim} S L(2 m) / H=\operatorname{dim} S L(2 m) / S p(2 m)+\operatorname{dim} \mathcal{O}^{\prime}=2 m^{2}-m-1+2 m=2 m^{2}+m-1$, where $\mathcal{O}^{\prime}$ is the $S p(2 m)$-orbit of $x_{\beta_{1}}(1)$, which has dimension $2 m$ (note that $\operatorname{dim} S L(2 m) / H=\operatorname{dim} S L(2 m) / S O(2 m)$, and $S O(2 m)$ is the centralizer of an outer involution of $S L(2 m)$ if the characteristic is not 2). Therefore $\operatorname{dim} S L(2 m) / H$ is the dimension of a Borel subgroup of $S L(2 m)$. As in the case when $n$ is even, we take $w=w_{0}$,

$$
x=\tau n_{\beta_{1}} \cdots n_{\beta_{m}} \in \tau w_{0} B .
$$

Then $x^{2}=1$ since $\tau\left(\beta_{k}\right)=\beta_{k}$ for each $k$, and for dimensional reasons, $x$ is conjugate to $\tau x_{\beta}(1)$.

We have established the information presented in Tables 17 and 18, where $w(\mathcal{O})=w_{0} w_{J}$.

TABLE 17. Outer involutions of $S L(2 m), m \geq 2, m$ even, $p=2$.

\begin{tabular}{|c||c|c|c|c|}
\hline $\mathcal{O}$ & $J$ & $w(\mathcal{O})$ & $x \in \mathcal{O} \cap \tau B w(\mathcal{O}) B$ & $\operatorname{dim} \mathcal{O}$ \\
\hline \hline$\tau$ & $\{1,3, \ldots, 2 m-1\}$ & $s_{\gamma_{1}} \cdots s_{\gamma_{m}}$ & $\tau x_{-\gamma_{1}}(1) \cdots x_{-\gamma_{m}}(1)$ & $2 m^{2}-m-1$ \\
\hline$\tau x_{\beta_{1}}(1)$ & $\emptyset$ & $w_{0}$ & $\tau n_{0}$ & $2 m^{2}+m-1$ \\
\hline
\end{tabular}

TABLE 18. Outer involutions of $S L(2 m), m \geq 3, m$ odd, $p=2$.

\begin{tabular}{|c||c|c|c|c|}
\hline $\mathcal{O}$ & $J$ & $w(\mathcal{O})$ & $x \in \mathcal{O} \cap \tau B w(\mathcal{O}) B$ & $\operatorname{dim} \mathcal{O}$ \\
\hline \hline$\tau$ & $\{1,3, \ldots, 2 m-1\}$ & $s_{\gamma_{1}} \cdots s_{\gamma_{m-1}}$ & $\tau x_{-\gamma_{1}}(1) \cdots x_{-\gamma_{m-1}}(1)$ & $2 m^{2}-m-1$ \\
\hline$\tau x_{\beta_{1}}(1)$ & $\emptyset$ & $w_{0}$ & $\tau n_{0}$ & $2 m^{2}+m-1$ \\
\hline
\end{tabular}

4.3. Type $D_{n}, n \geq 4$. To deal with $G$ of type $D_{n}$ we shall, as usual, consider $G=S O(2 n)$. Then the outer involutions of $G$ are obtained by conjugation with involutions of $O(2 n)$. Note that if $n=4$ and if $G$ is adjoint or simply-connected, then there are other outer involutions in Aut $G$. However, they are conjugate in Aut $G$ to involutions in $O(2 n)$.

Let $\tau$ be the involution of $O(2 n)$ inducing the graph automorphism of $S O(2 n)$, i.e. the graph automorphism acting trivially on $\left\langle X_{ \pm \alpha_{i}} \mid i \in\{1, \ldots, n-2\}\right\rangle$ and such that $x_{\alpha_{n-1}}(\xi) \leftrightarrow x_{\alpha_{n}}(\xi), x_{-\alpha_{n-1}}(\xi) \leftrightarrow x_{-\alpha_{n}}(\xi)$ for $\xi \in k$. 
The classes of involutions in $O(2 n) \backslash S O(2 n)$ correspond to partitions $2^{\ell} \oplus 1^{2 n-2 \ell}$ for $\ell \leq n$ with $\ell$ odd, with $\tau$ corresponding to $2 \oplus 1^{2 n-2}$. Let $\mathcal{O}_{\ell}$ be the class corresponding to $2^{\ell} \oplus 1^{2 n-2 \ell}$. From [28, $\left.\left.2.9 \mathrm{~b}\right)\right]$ we get

$$
\operatorname{dim} \mathcal{O}_{\ell}=\operatorname{dim} \mathcal{O}_{\ell, S p(2 n, \mathbb{C})}-2 n+\lambda_{1}^{*},
$$

where $\lambda_{1}^{*}=c_{1}+c_{2}=(2 n-2 \ell)+\ell=2 n-\ell$. Hence

$$
\operatorname{dim} \mathcal{O}_{\ell}=k(2 n-\ell+1)-2 n+2 n-\ell=\ell(2 n-\ell)
$$

for $\ell \leq n$ with $\ell$ odd.

Let

$$
\mu_{1}=e_{1}-e_{n}, \quad \nu_{1}=e_{1}+e_{n}, \quad w=s_{\mu_{1}} s_{\nu_{1}} .
$$

Then

$$
\ell(w)+\operatorname{rk}(1-\tau w)=2(n-1)+1=2 n-1=\operatorname{dim} \mathcal{O}_{1}
$$

and $\tau\left(\mu_{1}\right)=\nu_{1}$. We have

$$
n_{\nu_{1}} \tau n_{\nu_{1}}=\tau n_{\mu_{1}} n_{\nu_{1}}
$$

so that

$$
\tau n_{\mu_{1}} n_{\nu_{1}} \in \mathcal{O}_{1} \cap w B .
$$

To deal with the remaining classes, we put $m=\left[\frac{n}{2}\right]$ and

$$
\mu_{i}=e_{2 i-2}-e_{2 i-1}, \quad \nu_{i}=e_{2 i-2}+e_{2 i-1}, \quad w_{i}=s_{\mu_{1}} s_{\nu_{1}} \cdots s_{\mu_{i}} s_{\nu_{i}}
$$

for $i=2, \ldots, m$.

Arguing as above, we can prove that

$$
\ell\left(w_{\ell}\right)+\operatorname{rk}\left(1-\tau w_{\ell}\right)=\operatorname{dim} \mathcal{O}_{2 \ell-1}
$$

and

$$
\tau n_{\mu_{1}} n_{\nu_{1}} \cdots n_{\mu_{\ell}} n_{\nu_{\ell}} \in \mathcal{O}_{2 \ell-1} \cap w_{\ell} B
$$

for $\ell=2, \ldots, m$. In fact it is enough to count the number of Jordan blocks of dimension 2 in $\tau n_{\mu_{1}} n_{\nu_{1}} \cdots n_{\mu_{\ell}} n_{\nu_{\ell}}$ : in $\tau n_{\mu_{1}} n_{\nu_{1}}$ there is 1 , and in $n_{\mu_{i}} n_{\nu_{i}}$ there are 2 for each $i=2, \ldots, \ell$.

If $n$ is even, then there are $\frac{1}{2} n$ conjugacy classes of outer involutions, and we are done. In particular, the maximal one is $2^{n-1} \oplus 1^{2}$ and corresponds to $w=$ $s_{\mu_{1}} s_{\nu_{1}} \cdots s_{\mu_{m}} s_{\nu_{m}}=w_{0}=-1$.

If $n$ is odd, then there are $\frac{1}{2}(n+1)$ conjugacy classes of outer involutions: the maximal one is $\mathcal{O}_{n}=2^{n}$, which is the only one not in the previous list. We have

$$
\operatorname{dim} \mathcal{O}_{n}=n^{2}
$$

Let $n_{0}$ be any representative in $N$ of $w_{0}$ with $n_{0}$ of order 2 and commuting with $\tau$. Then $x=\tau n_{0}$ is an involution in $\tau w_{0} B$. Since

$$
\ell\left(w_{0}\right)+\operatorname{rk}\left(1-\tau w_{0}\right)=n^{2}-n+n=n^{2}
$$

by Theorem 4.1, we have $\operatorname{dim} B . x \geq n^{2}$. However, $x$ is an involution in $O(2 n) \backslash$ $S O(2 n)$, so that $\operatorname{dim} G . x \leq \operatorname{dim} \mathcal{O}_{n}=n^{2}$. Therefore $x$ lies in $\mathcal{O}_{n} \cap \tau w_{0} B$, and we are done.

Let $J_{\ell}=\{2 \ell, 2 \ell+1, \ldots, n\}$ for $\ell=1 \ldots, m$. We have established the information presented in Tables 19 and 20, where $w(\mathcal{O})=w_{0} w_{J}$. 
TABLE 19. Outer involutions of $D_{n}, n \geq 4, n=2 m, p=2$.

\begin{tabular}{|c||c|c|c|c|}
\hline $\mathcal{O}$ & $J$ & $w(\mathcal{O})$ & $x \in \mathcal{O} \cap \tau B w(\mathcal{O}) B$ & $\operatorname{dim\mathcal {O}}$ \\
\hline \hline $\begin{array}{c}2^{2 \ell-1} \oplus 1^{2 n-4 \ell+2} \\
\ell=1, \ldots, m-1\end{array}$ & $J_{\ell}$ & $s_{\mu_{1}} s_{\nu_{1}} \cdots s_{\mu_{\ell}} s_{\nu_{\ell}}$ & $\tau n_{\mu_{1}} n_{\nu_{1}} \cdots n_{\mu_{\ell}} n_{\nu_{\ell}}$ & $(2 \ell-1)(2 n-2 \ell+1)$ \\
\hline $2^{n-1} \oplus 1^{2}$ & $\emptyset$ & $w_{0}=s_{\mu_{1}} s_{\nu_{1}} \cdots s_{\mu_{m}} s_{\nu_{m}}$ & $\tau n_{\mu_{1}} n_{\nu_{1}} \cdots n_{\mu_{m}} n_{\nu_{m}}$ & $n^{2}-1$ \\
\hline
\end{tabular}

TABLE 20. Outer involutions of $D_{n}, n \geq 4, n=2 m+1, p=2$.

\begin{tabular}{|c||c|c|c|c|}
\hline $\mathcal{O}$ & $J$ & $w(\mathcal{O})$ & $x \in \mathcal{O} \cap \tau B w(\mathcal{O}) B$ & $\operatorname{dim} \mathcal{O}$ \\
\hline \hline $\begin{array}{c}2^{2 \ell-1} \oplus 1^{2 n-4 \ell+2} \\
\ell=1, \ldots, m\end{array}$ & $J_{\ell}$ & $s_{\mu_{1}} s_{\nu_{1}} \cdots s_{\mu_{\ell}} s_{\nu_{\ell}}$ & $\tau n_{\mu_{1}} n_{\nu_{1}} \cdots n_{\mu_{\ell}} n_{\nu_{\ell}}$ & $(2 \ell-1)(2 n-2 \ell+1)$ \\
\hline $2^{n}$ & $\emptyset$ & $w_{0}$ & $\tau n_{0}$ & $n^{2}$ \\
\hline
\end{tabular}

4.4. Type $E_{6}$. There are two (classes of) outer involutions, $\tau$ and $\tau x_{\beta_{1}}(1)$, where $\tau$ is the graph automorphism of $G$. We recall from 3.2 .1 that

$$
\begin{array}{ll}
\beta_{1}=(1,2,2,3,2,1), & \beta_{2}=(1,0,1,1,1,1), \\
\beta_{3}=(0,0,1,1,1,0), & \beta_{4}=(0,0,0,1,0,0) .
\end{array}
$$

Note that each $\beta_{i}$ is fixed by $\tau$.

Let us start with $\tau x_{\beta_{1}}(1)$. We have $K=C\left(\tau x_{\beta_{1}}(1)\right) \cong C_{F_{4}}\left(x_{\beta_{1}}(1)\right), \operatorname{dim} K=36$. Let $x=\tau n_{\beta_{1}} n_{\beta_{2}} n_{\beta_{3}} n_{\beta_{4}}$. Since $x$ is an involution in $\tau w_{0} B$, with $\ell\left(w_{0}\right)+\operatorname{rk}\left(1-\tau w_{0}\right)=$ $36+6=\operatorname{dim} E_{6} / K$, it follows that $\tau x_{\beta_{1}}(1) \sim x$.

To deal with $\tau$, we put $\delta_{1}=(1,1,2,2,1,1), \delta_{2}=(1,1,1,2,2,1)$. We have

$$
\operatorname{dim} E_{6} / F_{4}=26 \text {. }
$$

Note that $\tau\left(\delta_{1}\right)=\delta_{2}$ and

$$
\ell\left(s_{\delta_{1}} s_{\delta_{2}}\right)+\operatorname{rk}\left(1-\tau s_{\delta_{1}} s_{\delta_{2}}\right)=24+2=26 .
$$

In fact, here $J=\{2,3,4,5\}, w=s_{\delta_{1}} s_{\delta_{2}}=w_{0} w_{J}$.

We show that $\tau \sim \tau n_{\delta_{1}} n_{\delta_{2}}$. Let $g=x_{-\delta_{1}}(1)$. Then

$$
g \tau g^{-1}=\tau x_{-\delta_{1}}(1) x_{-\delta_{2}}(1) \in \tau B w B .
$$

Moreover, since $\left[\tau, x_{\delta_{1}}(1) x_{\delta_{2}}(1)\right]=1$, we get

$$
x_{\delta_{1}}(1) x_{\delta_{2}}(1) \tau x_{-\delta_{1}}(1) x_{-\delta_{2}}(1) x_{\delta_{1}}(1) x_{\delta_{2}}(1)=\tau n_{\delta_{1}} n_{\delta_{2}}
$$

and we are done. We have established the information presented in Table 21, where $w(\mathcal{O})=w_{0} w_{J}$.

TABle 21. Outer involutions of $E_{6}, p=2$.

\begin{tabular}{|c||c|c|c|c|}
\hline $\mathcal{O}$ & $J$ & $w(\mathcal{O})$ & $x \in \mathcal{O} \cap \tau B w(\mathcal{O}) B$ & $\operatorname{dim} \mathcal{O}$ \\
\hline \hline$\tau$ & $\{2,3,4,5\}$ & $s_{\delta_{1}} s_{\delta_{2}}$ & $\tau n_{\delta_{1}} n_{\delta_{2}}$ & 26 \\
\hline$\tau x_{\beta_{1}}(1)$ & $\emptyset$ & $w_{0}$ & $\tau n_{\beta_{1}} n_{\beta_{2}} n_{\beta_{3}} n_{\beta_{4}}$ & 42 \\
\hline
\end{tabular}

This completes the list of outer involutions of simple algebraic groups in characteristic 2. We have proved

Theorem 4.2. Let $G$ be a reductive connected algebraic group in characteristic 2, and let $\sigma$ be any involutory automorphism of $G$. Then the fixed point subgroup $H$ of $\sigma$ is a spherical subgroup of $G$.

We conclude with another application of Theorem 4.1. 
4.5. Type $G_{2}$ in $D_{4}$. We show briefly how one can prove that the subgroup of type $G_{2}$ in $D_{4}$ is spherical (in all characteristics). Let us assume $G$ of type $D_{4}$. Without loss of generality, we may assume $G$ is adjoint. Hence if we denote by $\varphi$ the graph automorphism of $G$ fixing $\alpha_{2}$ and mapping $\alpha_{1} \mapsto \alpha_{3} \mapsto \alpha_{4} \mapsto \alpha_{1}$, then the fixed point subgroup $K$ of $\varphi$ is of type $G_{2}$. Let $\delta_{1}=\alpha_{1}+\alpha_{2}+\alpha_{3}, \delta_{2}=\alpha_{1}+\alpha_{2}+\alpha_{4}$, $\delta_{3}=\alpha_{2}+\alpha_{3}+\alpha_{4}$, and let $w=w_{0} s_{2}=s_{\delta_{1}} s_{\delta_{2}} s_{\delta_{3}}$. We have

$$
\ell(w)+\operatorname{rk}(1-\varphi w)=14=\operatorname{dim} D_{4} / G_{2} .
$$

It remains to show that a $G$-conjugate of $\varphi$ lies in $\varphi B w_{0} s_{2} B \subseteq G:\langle\varphi\rangle$.

Let $g=x_{-\delta_{1}}\left(\xi_{1}\right) x_{-\delta_{2}}\left(\xi_{2}\right) x_{-\delta_{3}}\left(\xi_{3}\right)$. Then

$$
g \varphi g^{-1}=\varphi x_{-\delta_{1}}\left(-\xi_{1}-\xi_{3}\right) x_{-\delta_{2}}\left(-\xi_{2}-\xi_{1}\right) x_{-\delta_{3}}\left(-\xi_{3}+\xi_{2}\right) .
$$

If we choose $\xi_{1}, \xi_{2}, \xi_{3}$ such that $\xi_{1}+\xi_{3}, \xi_{2}+\xi_{1}$ and $-\xi_{3}+\xi_{2}$ are non-zero, then $g \varphi g^{-1} \in \varphi B w_{0} s_{2} B$, and we are done.

Remark 4.3. If the characteristic is zero, we may apply the arguments in 11 . Since $T^{\varphi w}=H_{\alpha_{2}}$ is connected, it follows that, in the simply-connected case, the monoid $\lambda\left(D_{4} / G_{2}\right)$ of $B$-weights in $k\left[D_{4} / G_{2}\right]$ is generated by $\omega_{1}, \omega_{3}, \omega_{4}$, since $G_{2}$ is connected and has no non-trivial characters, so that the monoid $\lambda\left(D_{4} / G_{2}\right)$ is free (and it contains $(1-\varphi w) P^{+}$, which is the monoid generated by $\omega_{1}+\omega_{3}, \omega_{1}+\omega_{4}$, $\left.\omega_{3}+\omega_{4}, \omega_{1}+\omega_{3}+\omega_{4}\right)$ (see also [20]).

\section{ACKNOWLEDGEMENTS}

The author wishes to thank the referee for his helpful suggestions.

\section{REFERENCES}

[1] A. V. AlekseevskiI, Component groups of centralizers of unipotent elements in semisimple algebraic groups, Trudy Tbiliss. Mat. Inst. Razmadze Akad. Nauk. Gruzin SSR 62, 5-27 (1979), Lie groups and invariant theory, Amer. Math. Soc. Transl. Ser. 2, 213, Amer. Math. Soc. Providence, RI (2005). MR557505 (81k:20063)

[2] M. Aschbacher, G.M. Seitz, Involutions in Chevalley groups over fields of even order, Nagoya Math. J. 63, 1-91 (1976). MR0422401 (54:10391)

[3] N. Bourbaki, Éléments de Mathématique. Groupes et Algèbres de Lie, Chapitres 4,5, et 6, Masson, Paris (1981). MR647314 (83g:17001)

[4] J. Brundan, Dense orbits and double cosets, Algebraic groups and their representations (Cambridge, 1997), 259-274, NATO Adv. Sci. Inst. Ser. C Math. Phys. Sci., 517, Kluwer Acad. Publ., Dordrecht (1998). MR.1670774 (99k:20090)

[5] N. Cantarini, G. Carnovale, M. Costantini, Spherical orbits and representations of $\mathcal{U}_{\varepsilon}(\mathfrak{g})$, Transformation Groups 10, No. 1, 29-62 (2005). MR2127340 (2005m:17020)

[6] G. Carnovale, Spherical conjugacy classes and involutions in the Weyl group, Math. Z. 260(1), 1-23 (2008). MR2413339 (2009d:20091)

[7] G. Carnovale, Spherical conjugacy classes and Bruhat decomposition, Ann. Inst. Fourier Grenoble 59, No. 6, 2329-2357 (2009). MR2640922 (2011c:20094)

[8] G. Carnovale, A classification of spherical conjugacy classes in good characteristic, Pacific J. Math. 245, No. 1, 25-45 (2010). MR2602680 (2011c:20095)

[9] M. Costantini On the lattice automorphisms of certain simple algebraic groups, Rend. Sem. Mat. Univ. Padova 90, 141-157 (1993). MR.1257137 (95h:20034)

[10] M. Costantini The lattice automorphisms of simple algebraic groups over $\overline{\mathbb{F}}_{2}$, Manuscripta Math. 91, 1-16 (1996). MR1404414 (98b:20068)

[11] M. Costantini, On the coordinate ring of spherical conjugacy classes, Math. Z. 264, No. 2, 327-359 (2010). MR 2574980 (2010k:20071)

[12] R. W. Carter, Simple Groups of Lie Type, John Wiley (1989). MR.1013112 (90g:20001)

[13] R. W. CARTER, Finite Groups of Lie Type, John Wiley (1985). MR794307 (87d:20060) 
[14] K.Y. Chan, J.-H. Lu, S.K.M. To, On intersections of conjugacy classes and Bruhat cells, preprint ArXiv:0906.2254v1.

[15] R. Fowler, G. Röhrle, Spherical nilpotent orbits in positive characteristic, Pacific J. Math. 237, 241-286 (2008). MR2421122 (2009f:14095)

[16] W.H. Hesselink, Nilpotency in classical groups over a filed of characteristic 2, Math. Z. 54, 165-181 (1979). MR525621 (82d:14030)

[17] J.E. Humphreys, Linear Algebraic Groups, Springer-Verlag, New York (1995). MR0396773 $(53: 633)$

[18] N. IWAHORI, Centralizers of involutions in finite Chevalley groups, In: "Seminar on algebraic groups and related finite groups". LNM 131, 267-295, Springer-Verlag, Berlin, Heidelberg, New York (1970). MR0258945(41:3590)

[19] F. KNop, On the set of orbits for a Borel subgroup, Comment. Math. Helv. 70, 285-309 (1995). MR:1324631 (96c:14039)

[20] M. KRÄMER, Sphärische Untergruppen in kompakten zusammenhangenden Liegruppen, Compositio Math. 38 (1979), 129-153. MR528837(80f:22011)

[21] R. Lawther, M.W. Liebeck, G.M. Seitz, Fixed point spaces in actions of exceptional algebraic groups, Pacific J. Math. 205, 339-391 (2002). MR.1922739 (2003g:20080)

[22] G. Lusztig, Remarks on Springer's representations, Represent. Theory 13, 391-400 (2009). MR2540702 (2011a:17010)

[23] T. MATSUKI, The orbits of affine symmetric spaces under the action of minimal parabolic subgroups, J. Math. Soc. Japan 31, 331-357 (1979). MR.527548 (81a:53049)

[24] D. Panyushev, Complexity and nilpotent orbits, Manuscripta Math. 83, 223-237 (1994). MR1277527 (95e:14039)

[25] D. Panyushev, On spherical nilpotent orbits and beyond, Ann. Inst. Fourier, Grenoble 49(5), 1453-1476 (1999). MR1723823 (2000i:14072)

[26] D. Panyushev, Some amazing properties of spherical nilpotent orbits, Math. Z. 245, 557-580 (2003). MR 2021571 (2004j:14051)

[27] G.M. Seitz, Double cosets in algebraic groups, Algebraic groups and their representations (Cambridge, 1997), 214-257, NATO Adv. Sci. Inst. Ser. C Math. Phys. Sci., 517, Kluwer Acad. Publ., Dordrecht (1998). MR1670773 (99k:20096)

[28] J.N. Spaltenstein, Classes unipotentes et sous-groupes de Borel, LNM 946, Springer-Verlag, Berlin, Heidelberg, New York (1982). MR672610(84a:14024)

[29] T.A. Springer, Some results on algebraic groups with involutions, Algebraic groups and related topics (Kyoto/Nagoya, 1983), 525-543, Adv. Stud. Pure Math. 6, North-Holland, Amsterdam (1985). MR803346 (86m:20050)

[30] T.A. Springer, Linear Algebraic Groups, Second Edition, Progress in Mathematics 9, Birkhäuser (1998). MR1642713 (99h:20075)

[31] R. Steinberg, Lectures on Chevalley groups, Yale University (1967). MR0466335 (57:6215)

[32] T. Vust, Opération de groupes réductifs dans un type de cônes presques homogènes, Bull. Soc. Math. France 102, 317-333 (1974). MR0366941 (51:3187)

[33] G.E. WALL, On the conjugacy classes in the unitary, symplectic and orthogonal groups, J. Austral. Math. Soc. 3, 62-89 (1963). MR 0150210(27:212)

Dipartimento di Matematica Pura ed Applicata, Università degli Studi di Padova, Torre Archimede, via Trieste 63, 35121 Padova, Italy

E-mail address: costantini@math.unipd.it 